\title{
Lattice disorder and oxygen migration pathways in pyrochlore and defect-fluorite oxides
}

Frederick P. Marlton ${ }^{\dagger}$, Zhaoming Zhang ${ }^{\ddagger}$, Yuanpeng Zhang ${ }^{\S}$, Thomas E. Proffen ${ }^{\S}$, Chris D. Ling $^{\dagger}$, Brendan J. Kennedy ${ }^{*} \dagger$

†School of Chemistry, The University of Sydney, Sydney, NSW 2006, Australia

¥Australian Nuclear Science and Technology Organisation, Lucas Heights, NSW 2234, Australia

§Neutron Scattering Division, Oak Ridge National Laboratory, Oak Ridge, TN 37831, USA 


\section{Supplementary 1, Structures and results}

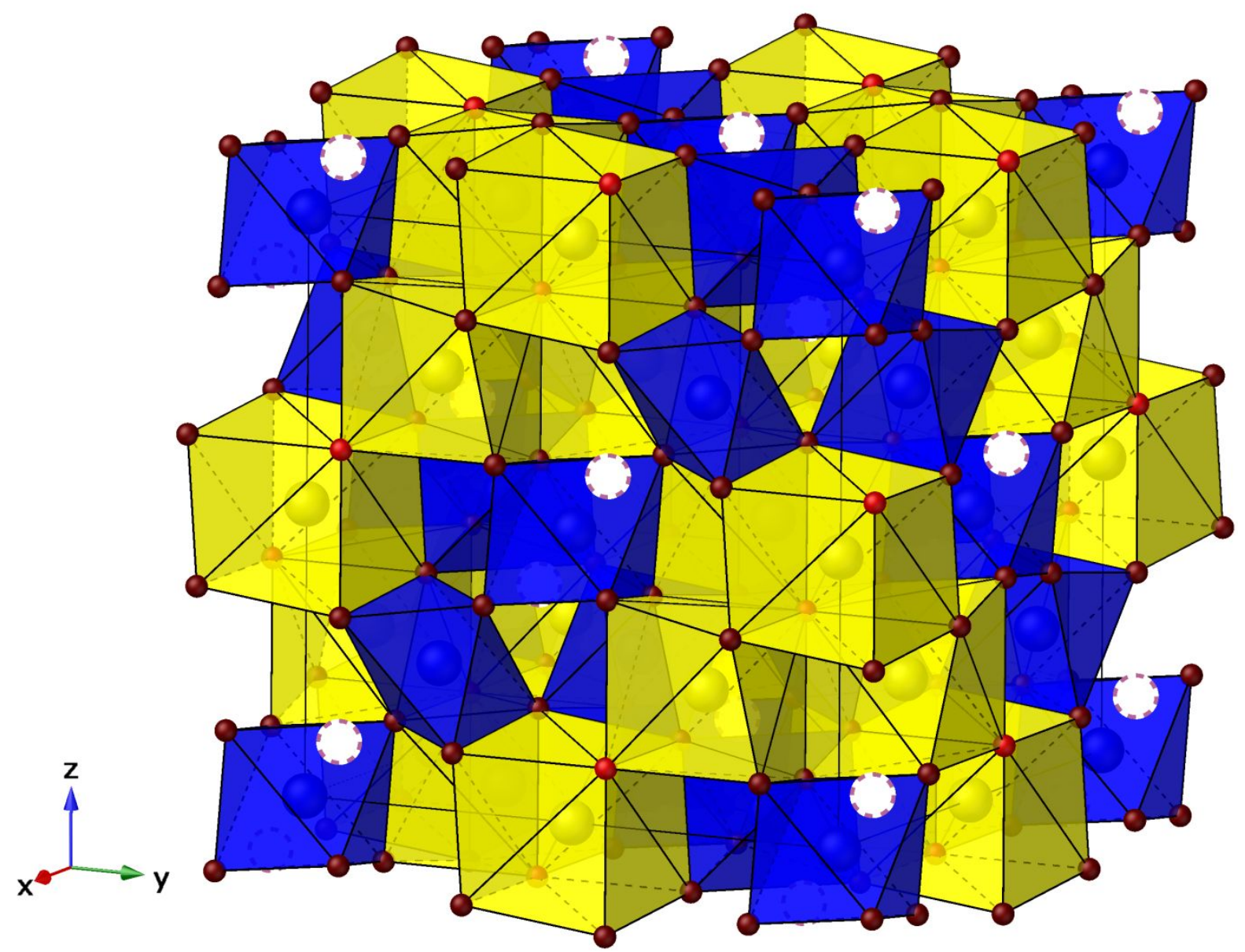

Figure S1: Cation sublattice of the pyrochlore $\mathrm{A}_{2} \mathrm{~B}_{2} \mathrm{O}_{7}$ structure. $A$ and $B$ sites are repreesented by yellow and blue spheres, respectively. Red spheres are oxygen atoms, with the light and dark representing the $8 b$ and $48 f W y c k o f f$ sites, respectively. White circles with purple dashed lines represent the vacant 8 a site. 


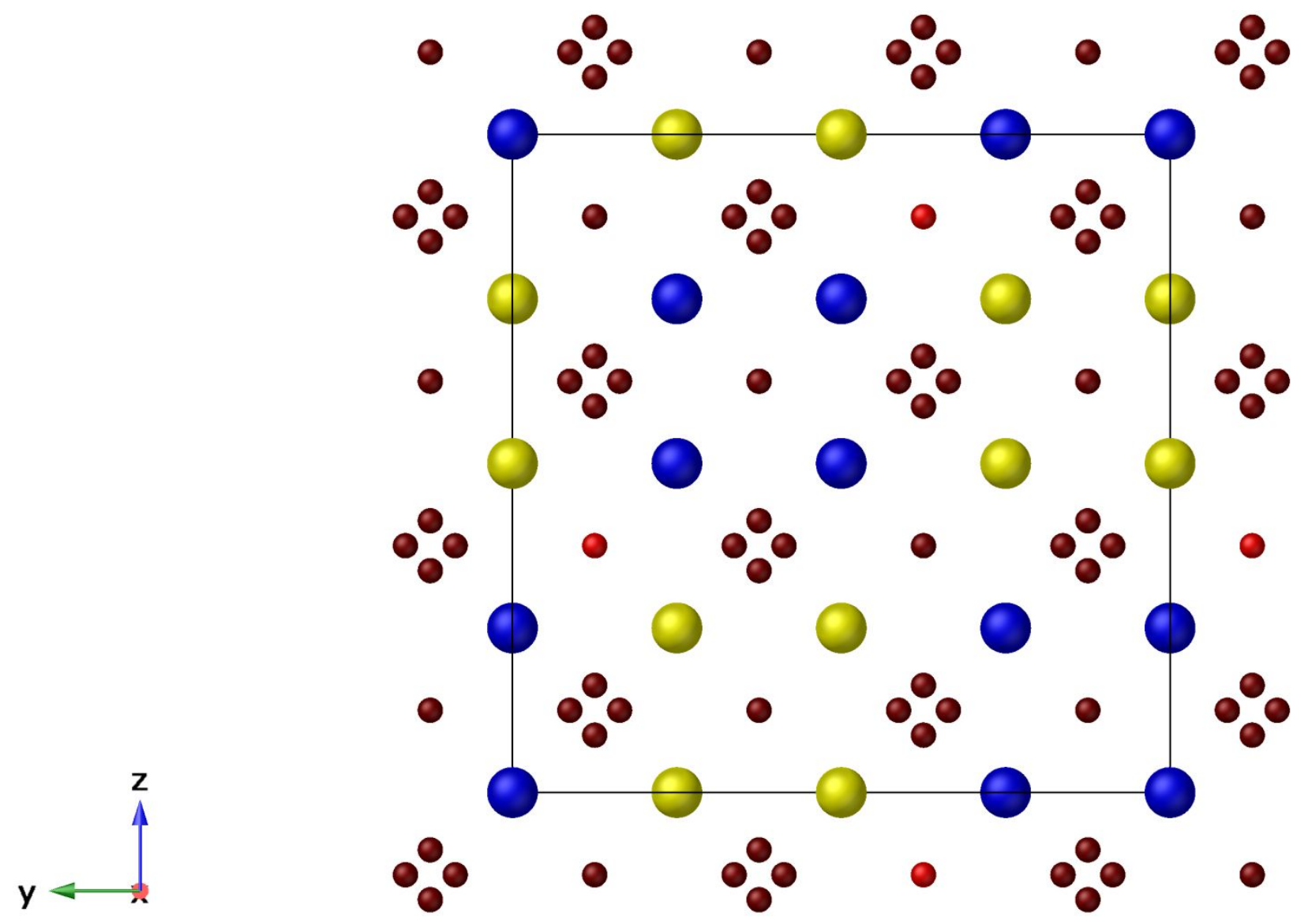

Figure S2: Structure from Figure 1 viewed down the a-axis without drawing polyhedra or vacancies. 


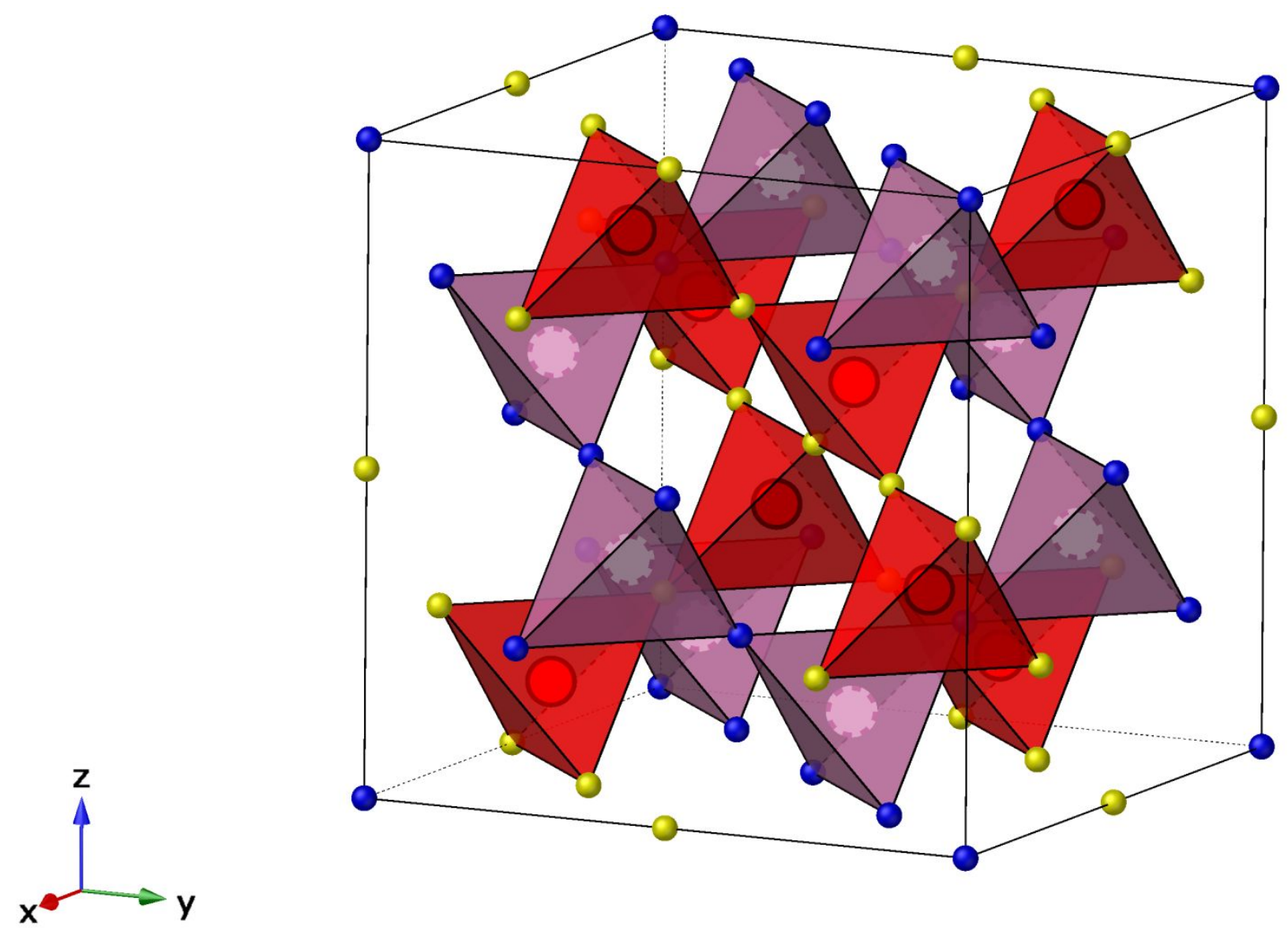

Figure S3: Anion sublattice of the pyrochlore structure with the $48 f$ oxygens ommitted. Red tetrahedra are formed by the A-site cations around the $8 b$ site. Purple tetrahedra are formed by the B-site cations around the vacant $8 a$ site. 


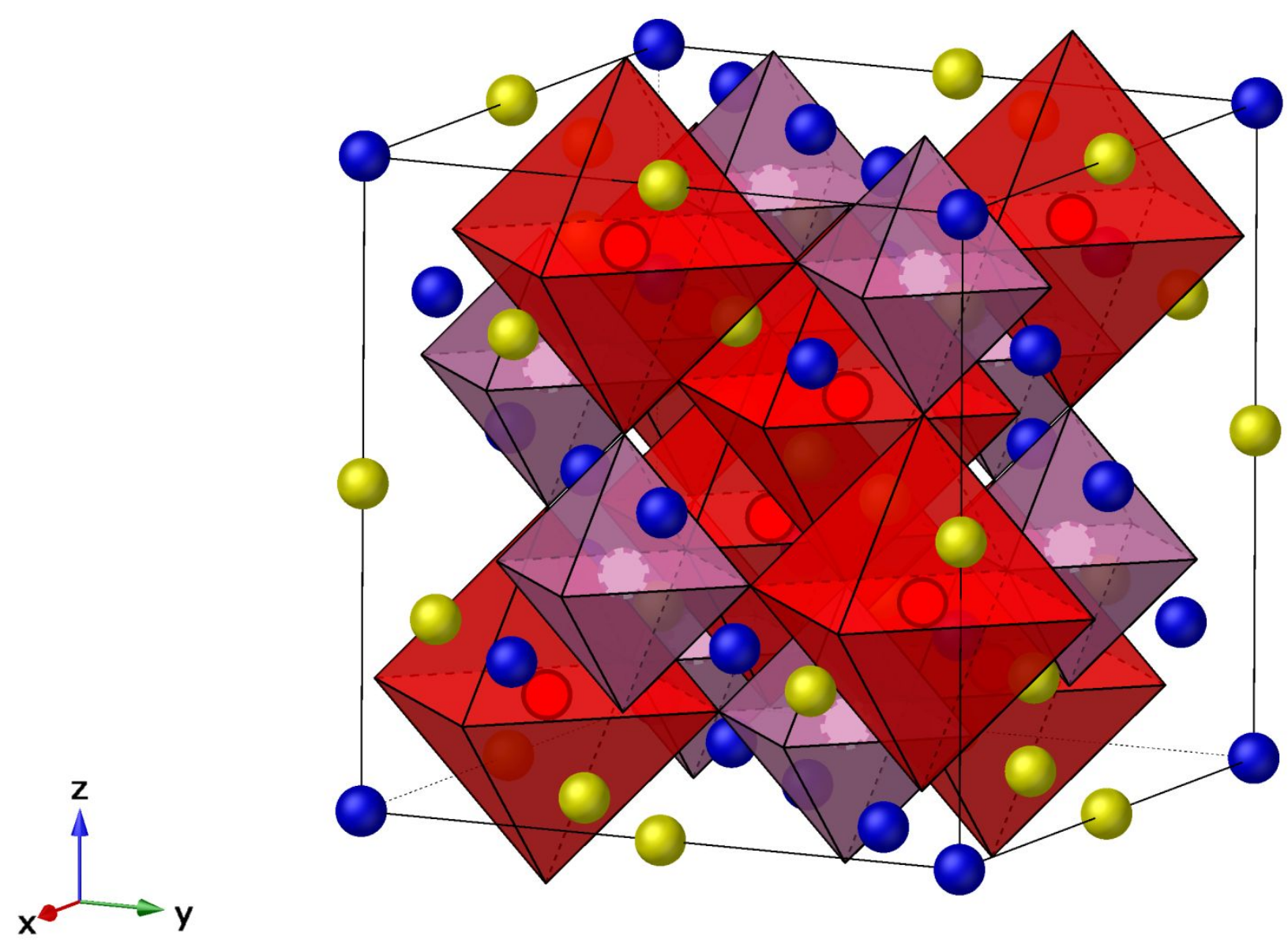

Figure S4: Representation of the anion sublattice of the pyrochlore structure. Interstitial non-bonding octahedra are formed by the $48 f$ oxygens around the $8 b$ (solid red circle) and $8 a$ (dashed white circle) sites. Yellow and blue spheres correspond to the $A$ and $B$ site cations, respecitvely. 


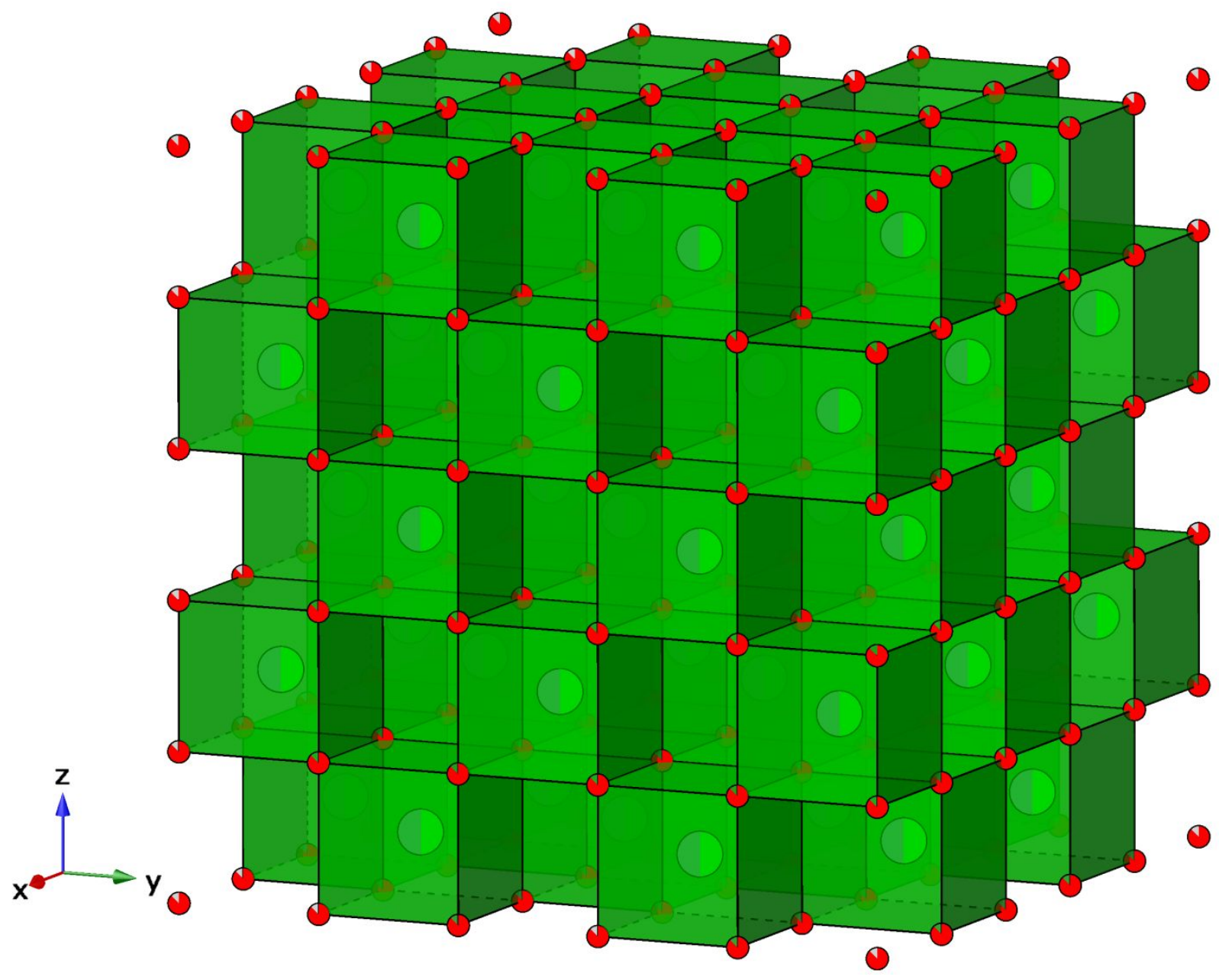

Figure S5: Disordered fluorite structure. Mixed A and B cations are represented by the mixed grey/green circles. Mixed vacancy and oxygen atoms are represented by the mixed red/grey circles. 


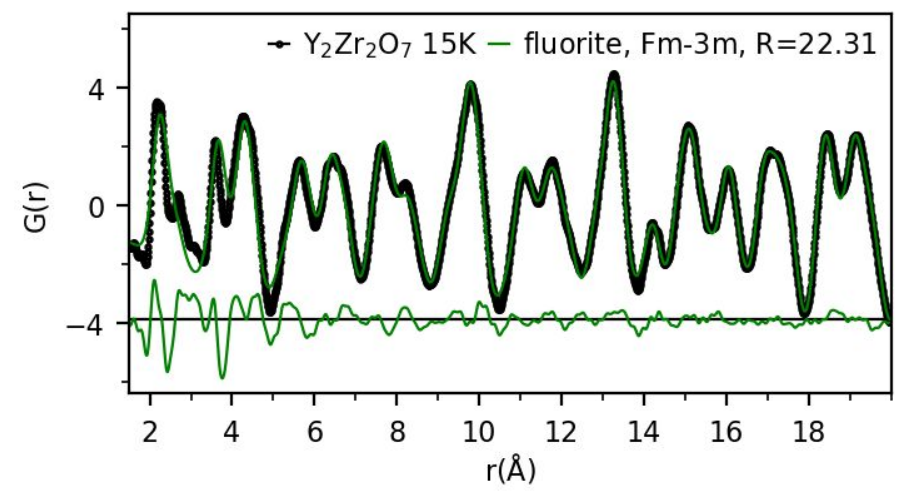

Figure S6: Small-box modelling fit to the $\mathrm{Y}_{2} \mathrm{Zr}_{2} \mathrm{O}_{7} 15 \mathrm{~K}$ data using the defect-fluorite $(\mathrm{Fm} \overline{3} \mathrm{~m})$ structural model.

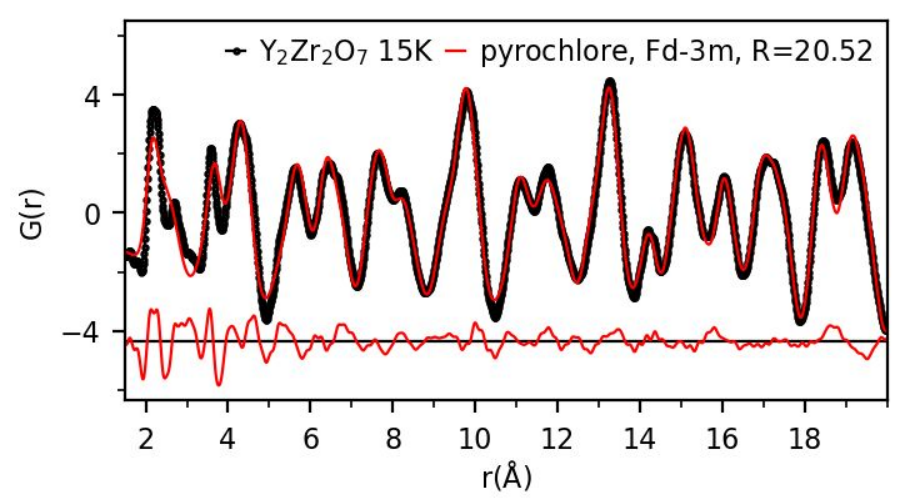

Figure S7: Small-box modelling fit to the $\mathrm{Y}_{2} \mathrm{Zr}_{2} \mathrm{O}_{7} 15 \mathrm{~K}$ data using the pyrochlore $(\mathrm{Fd} \overline{3} \mathrm{~m})$ structural model.

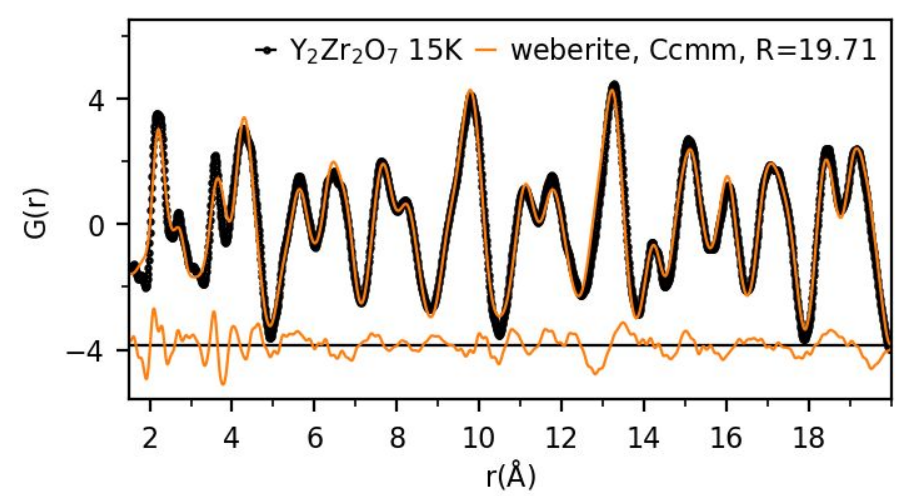

Figure S8: Small-box modelling fit to the $\mathrm{Y}_{2} \mathrm{Zr}_{2} \mathrm{O}_{7} 15 \mathrm{~K}$ data using the weberite $(\mathrm{Ccmm})$ structural model. 


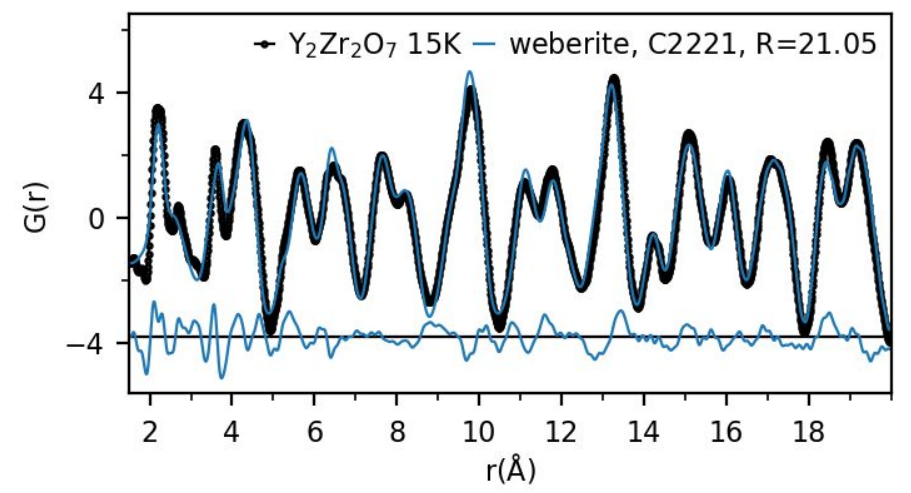

Figure S9: Small-box modelling fit to the $\mathrm{Y}_{2} \mathrm{Zr}_{2} \mathrm{O}_{7} 15 \mathrm{~K}$ data using the weberite (C222 $2_{1}$ ) structural model.

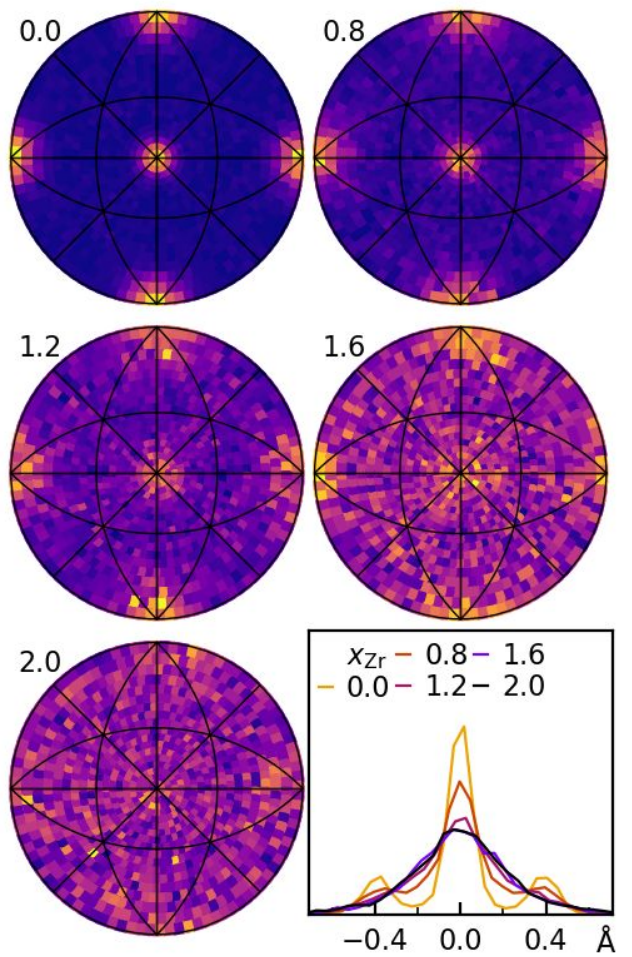

Figure S10: Alternative version of Figure $2 a$ in the main text in which each stereographic projection is plotted on an independent color scale. 


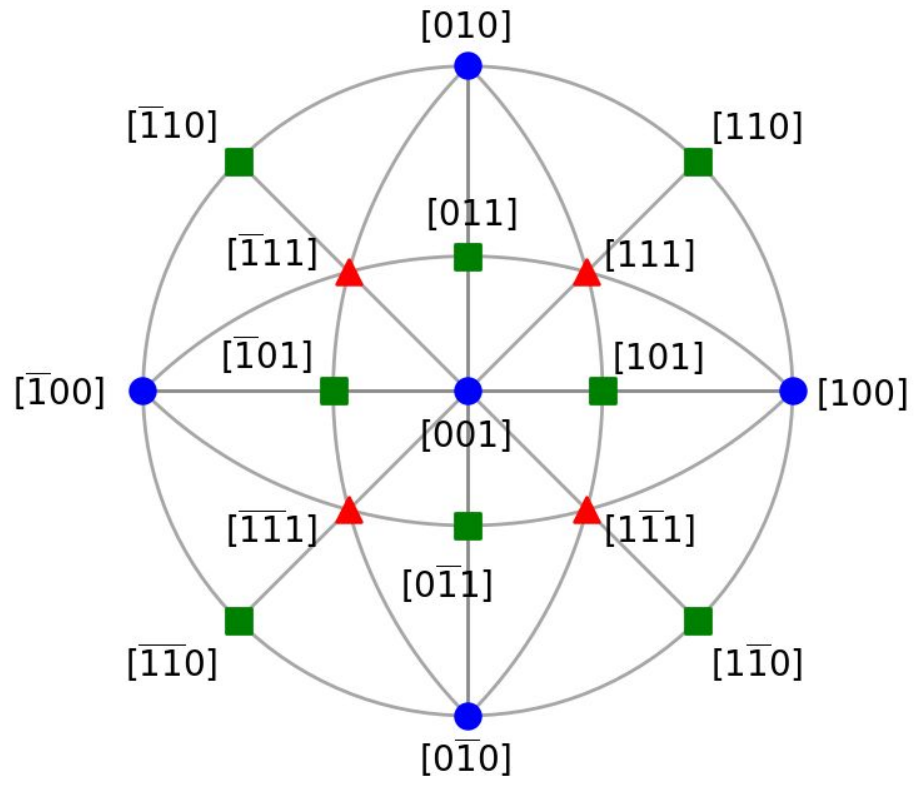

Figure S11: Reference directions for stereographic projections viewed down [001]. 


\section{Supplementary 2, RMC strategy}

Fitting the PDF data by small-box modelling in Topas ${ }^{1-2}$ was conducted using the notation from Equation 43 (referred here as $G(r)$ ) in Keen, D. $(2001)^{3}$, while fitting the PDF data by big-box modelling in RMCProfile 4 was conducted using the notation from Equation 10 (referred here as $g(r)$ ) in Keen, $D$. $(2001)^{3}$. RMCProfile allows for a variety of real- and reciprocal-space notations to be fit. The $g(r)$ notation places greater emphasis on the nearest neighbor features in the local structure. Hence, the atom moves in the RMC algorithm, which are partly determined by the PDF $\chi^{2}$, will most strongly reflect the local-scale features. The long-range features are not neglected, as they are determined by simultaneously fitting the $F(Q)$ data.

A common strategy implemented in RMC modelling is to repeat refinements to assess any variation in the results. However, in crystalline materials, a well-defined average structure can constrain the configurational space such that the same refinement parameters and starting configuration are likely to converge to similar results. In this case, refinements with variations in the parameters and starting configuration were conducted to allow a greater variety of configurational space to be explored and assess the robustness of the RMC model. The different refinement strategies tested were based upon two variations. The first variation involved the starting configuration, which was varied by cation coordination numbers and achieved by changing the positions of the vacancies ( $\left.\mathrm{V}_{0}\right)$ (Figure $\left.\mathrm{S} 12\right)$. For the ideal pyrochlore, the vacancies occupy the $8 a$ Wyckoff site in the $F d \overline{3} m$ structure (labelled n-pyro in

figures below). If the structure is 7-coordinate on all the $A$ - and $B$ - sites, then the vacancies occupy ${ }^{1} / 6$ of the $48 \mathrm{f}$ site (labelled 7-pyro in figures below). Another configuration is the 'inverse pyrochlore', where the vacancies occupy the $8 b$ site, resulting in the $A$ - and $B$-sites being 6 - and 8 -coordinate, respectively (labelled i-pyro in figures below). RMC refinements with these three coordination configurations fixed were tested on the $\mathrm{Y}_{2} \mathrm{Zr}_{2} \mathrm{O}_{7}$ end-member (Figure S14). The coordination of these cations was then altered by allowing swapping between the vacancies and oxygens in the RMC refinement. Ideally, irrespective of the starting configuration, the final configuration should be the same or very similar. This is particularly important for $\mathrm{Y}_{2} \mathrm{Sn}_{2} \mathrm{O}_{7}$ where the final configuration is well known.

The second variation that affects the refinement is the minimum distance constraint between vacancies. Distance constraints are commonly used in RMCProfile refinements to prevent unphysical moves being accepted. However, this can also affect the freedom of motion of the vacancies (Figure S13). Hence, for each of the starting configurations, refinements were tested with different $\mathrm{V}_{0}-\mathrm{V}_{\mathrm{O}}$ minimum distances. These correspond to allowing two vacancies around a cation to be separated along the $\langle 100\rangle,\langle 110\rangle$ and $<111>$ directions, as illustrated in Figure S13.

For each of the starting configurations, there are $3 \mathrm{~V}_{0}-\mathrm{V}_{0}$ distance constraints. However, the $<111>$ constraint cannot be tested for the initial 7-coordinate configuration as any vacancy movement violates this constraint, resulting in an fixed coordination state. Hence, there are a total of 8 refinement strategies being implemented on each composition. Each of these different refinement strategies allows a greater variety of configurational space to be explored and tests the robustness of the RMC modelling. To assess the physical nature of the refinement, statistics on the cation coordination and interatomic distances were extracted, focusing on the end-members of $\mathrm{Y}_{2} \mathrm{Sn}_{2} \mathrm{O}_{7}$ and $\mathrm{Y}_{2} \mathrm{Zr}_{2} \mathrm{O}_{7}$. 
The quality of a single RMC refinement for each of the strategies can be observed in Figure S15 - Figure S24, where in all cases a good fit was achieved. It was found that irrespective of the refinement strategy, the statistics of the structural distortions were very similar, which can be seen in Figure S25 - Figure S38. Figure S25 - Figure S26 show partial PDFs, Figure S27 - Figure S34 show atomic displacements, Figure S35 - Figure S36 show bond angles and Figure 37 - Figure S38 show average M-O polyhedra distances.

The main deviation between the methodologies is in the statistics regarding anion ordering as shown in Figure S39 - Figure S40. In these Figures it can be seen that the initial configuration had very little impact on the final result. The main impact came from the $V_{0}-V_{O}$ distance constraints. Figure S39 shows the location of the vacancies with respect to the Wyckoff sites. It can be seen that the $\langle 111\rangle V_{0}$ constraint results in some vacancies occurring in the $8 b$ site, which is unlikely given this has not been observed in the literature and represents a large displacement distance (Figure S4). This means that the $<111>V_{0}$ constraint is restricting the vacancy movement in the refinement too much. The $\langle 100\rangle$ and $\langle 110\rangle V_{O}$ constraints result in much more physically reasonable vacancy distributions in the Wyckoff sites. However, the $\langle 100\rangle V_{O}$ constraint also results in higher percentages of unphysical cation coordination, such as the 5-coordinate B-sites (Figure S40). It can also be observed in Figure $\$ 42$ that the $<100\rangle V_{0}$ constraint results in the highest percentage of vacancies separated along the $\langle 100\rangle$ directions for 6 -coordinate B-sites in $\mathrm{Y}_{2} \mathrm{Zr}_{2} \mathrm{O}_{7}$. Given this, the $\langle 110\rangle \mathrm{V}_{0}$ constraint gives the most physically reasonable constraint as it results in the best balance between degrees of freedom and physically reasonable results. This results in a significant percentage of vacancies separated along the $<110\rangle$ directions in the 6-coordinate $\mathrm{B}$-site $\mathrm{Y}_{2} \mathrm{Zr}_{2} \mathrm{O}_{7}$ (Figure S42). However, there is the possibility that such vacancy separations could occur transiently in the structure.

A future development in RMCProfile may involve the implementation of a "soft" distance-window constraint. The current distance-window constraint is "hard", meaning that moves are completely rejected when they violate the minimum or maximum pair distances. A "soft" constraint could allow these moves, but the overall goal is to minimize them. This is particularly important for highly disordered structures, where unphysical pair distances may need to occur temporarily in the progress of a refinement. This is also important for ordered structures, which is demonstrated by the result in Figure S39 for the $\mathrm{Y}_{2} \mathrm{Sn}_{2} \mathrm{O}_{7}$ composition with a 7-ccordinate initial configuration and the $\langle 110\rangle \mathrm{V}_{\mathrm{O}}$ constraint. In this case, the refined number of vacancies in the $48 f$ and $8 a$ sites is significantly different from all other initial configurations with the $<100>$ or $\langle 110\rangle V_{0}$ constraint. In the $7 C N$ configuration, the vacancies occupy $48 f$ sites. If a single vacancy were to move to another $48 f$ site at the start of the refinement, it would make no difference to the $\chi^{2}$, making it unlikely to be accepted. If the vacancy were to move to the $8 a$ or $8 b$ site, it would violate the $<110\rangle V_{0}$ constraint. Hence, the refinement is unable to change the vacancy configuration at the start. It is only after some atom displacements that this becomes possible, but it is still unable to reach the similar values of the other results. The "soft" distance-window constraint could allow for the desired configuration to be achieved.

In the RMC refinement, vacancies have a relatively small impact. They make up $12.5 \%$ of all possible oxygen sites and $8.3 \%$ of all atoms. They are also artificial, given that the structure is completely charge balanced. They contribute small amounts of intensity reduction to the PDF resulting in a small impact on the $\chi^{2}$ in the RMC algorithm. This can be observed in Figure S14 where the difference curve is only marginally different for the different refinements with fixed coordination. The difference curve is greatest for the "inverse pyrochlore". The algorithm in RMCProfile also has very few chemical constraints, with only the BVS being implemented here, which has no directional aspect to it as it only 
considers the number of bonded atoms and their bond length, but not their 3D location. Additionally, RMCProfile assesses atom moves one at a time. Although it does accept a fraction of bad moves, it may be necessary for a vacancy to make a particular sequence of bad moves before reaching its best final configuration, which is unlikely in the current algorithm. Furthermore, vacancy swapping would instantly alter the local energy landscape resulting in subsequent local relaxations or distortions. Therefore, an optimal fitting strategy would include subsequent moves around a swapped anion site, which is not currently implemented, further justifying the extra care that has been implemented here. There is also no mechanism to account for noise in the data and hence, the refinement, with its large degrees of freedom, can fit some of these features.

Given this, the results we have observed here are very positive and indicate a robust modelling methodology. This is because the initial configuration has very little impact on the final configuration, with the $\mathrm{V}_{\mathrm{O}}-\mathrm{V}_{\mathrm{O}}$ distance constraint having the biggest impact. Additionally, unphysical results make up a small percentage of the results, which is also demonstrated in Figure $4 \mathrm{c}$ of the main text. In the main text, the results from refinements with the $\langle 110\rangle V_{0}-V_{0}$ distance constraint and the ideal pyrochlore starting configuration was used. This is because the $\langle 110\rangle$ constraint provides the best balance between freedom of motion and unphysical results. 


\section{RMC refinement strategy $-\mathrm{Y}_{2} \mathrm{Zr}_{2} \mathrm{O}_{7}$ Initial configuration}
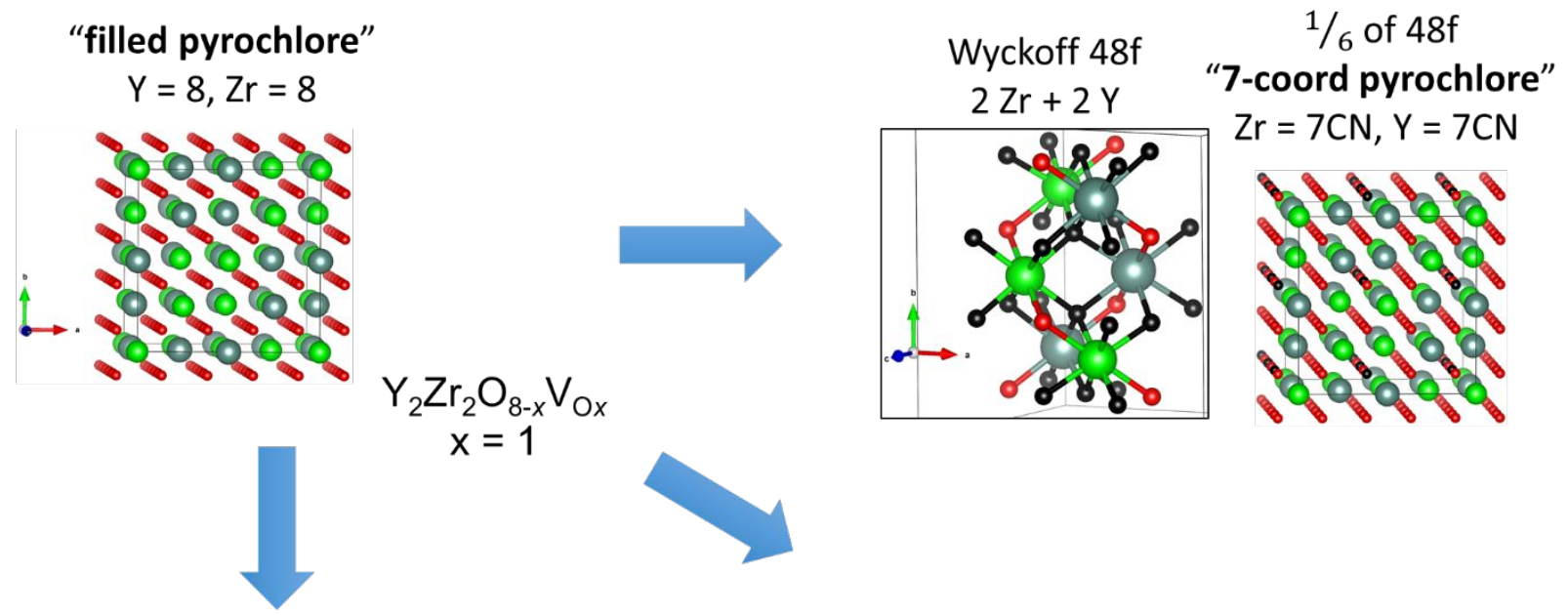

Wyckoff 8a

"Normal pyrochlore"

$4 \mathrm{Zr}$

$\mathrm{Zr}=6 \mathrm{CN}, \mathrm{Y}=8 \mathrm{CN}$
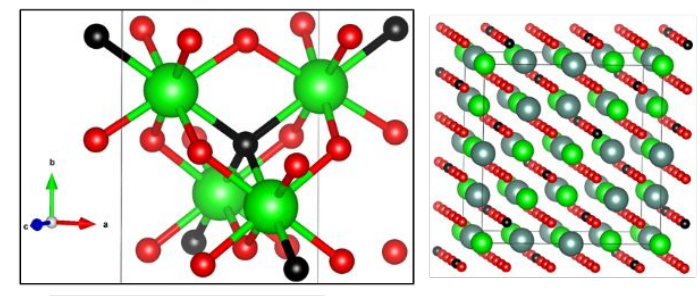

Wyckoff 8b

"Inverse pyrochlore"

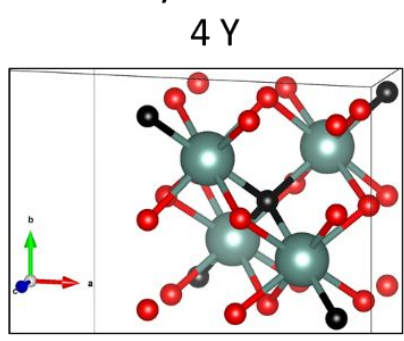

$\mathrm{Zr}=8 \mathrm{CN}, \mathrm{Y}=6 \mathrm{CN}$

Figure S12: Illustration of the way in which different starting configurations were generated for big box modelling by changing the positions of the vacancies $\left(V_{0}\right)$. 


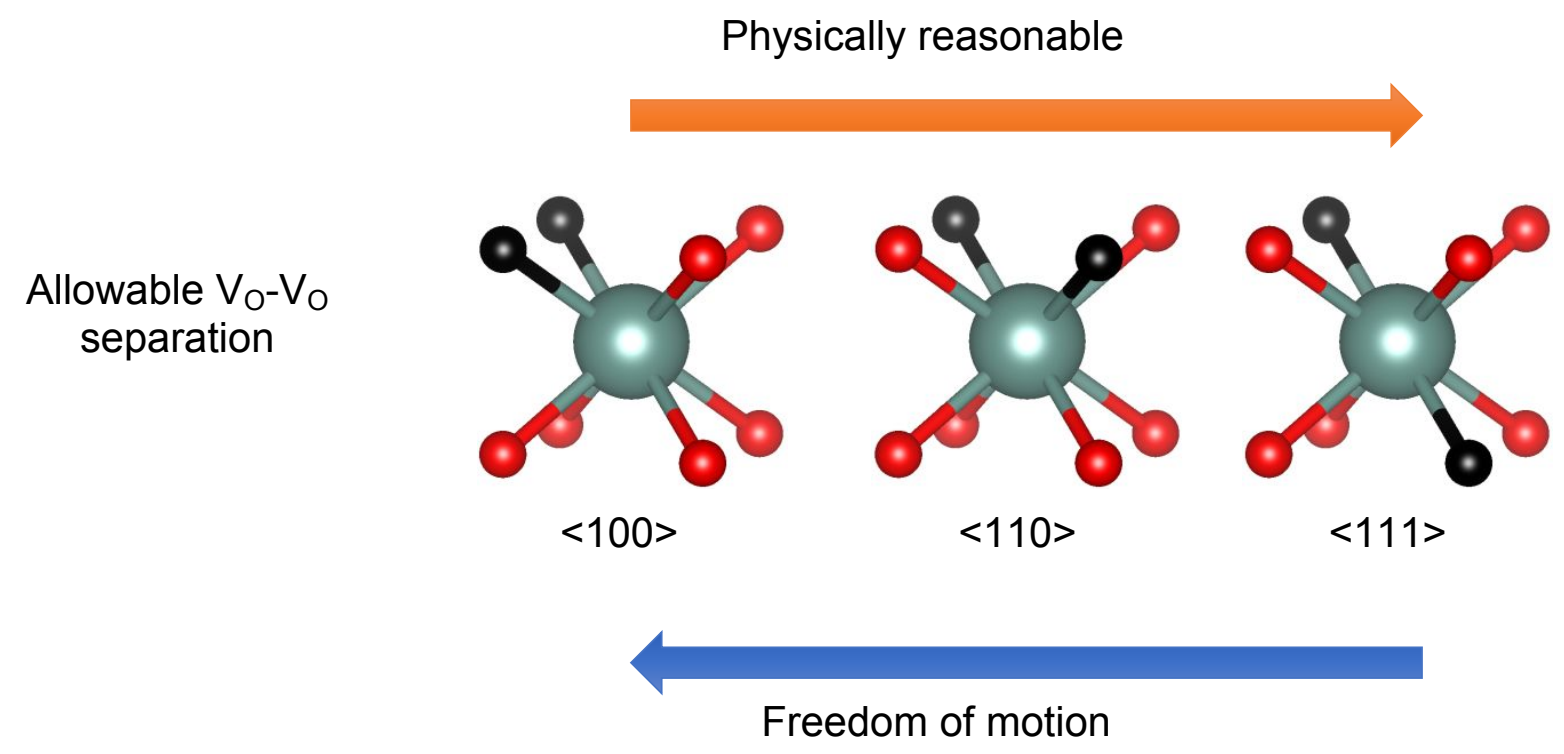

Figure S13: Illustration of the impact of $V_{O}-V_{O}$ distance constraints on the big box model. 


\section{Fits}

\section{Fixed cation coordinations}

The labels n-pyro, 7-pyro and i-pyro refer to the ideal, 7-coordinate and "inverse" pyrochlore starting configurations of the vacancies as discussed above.

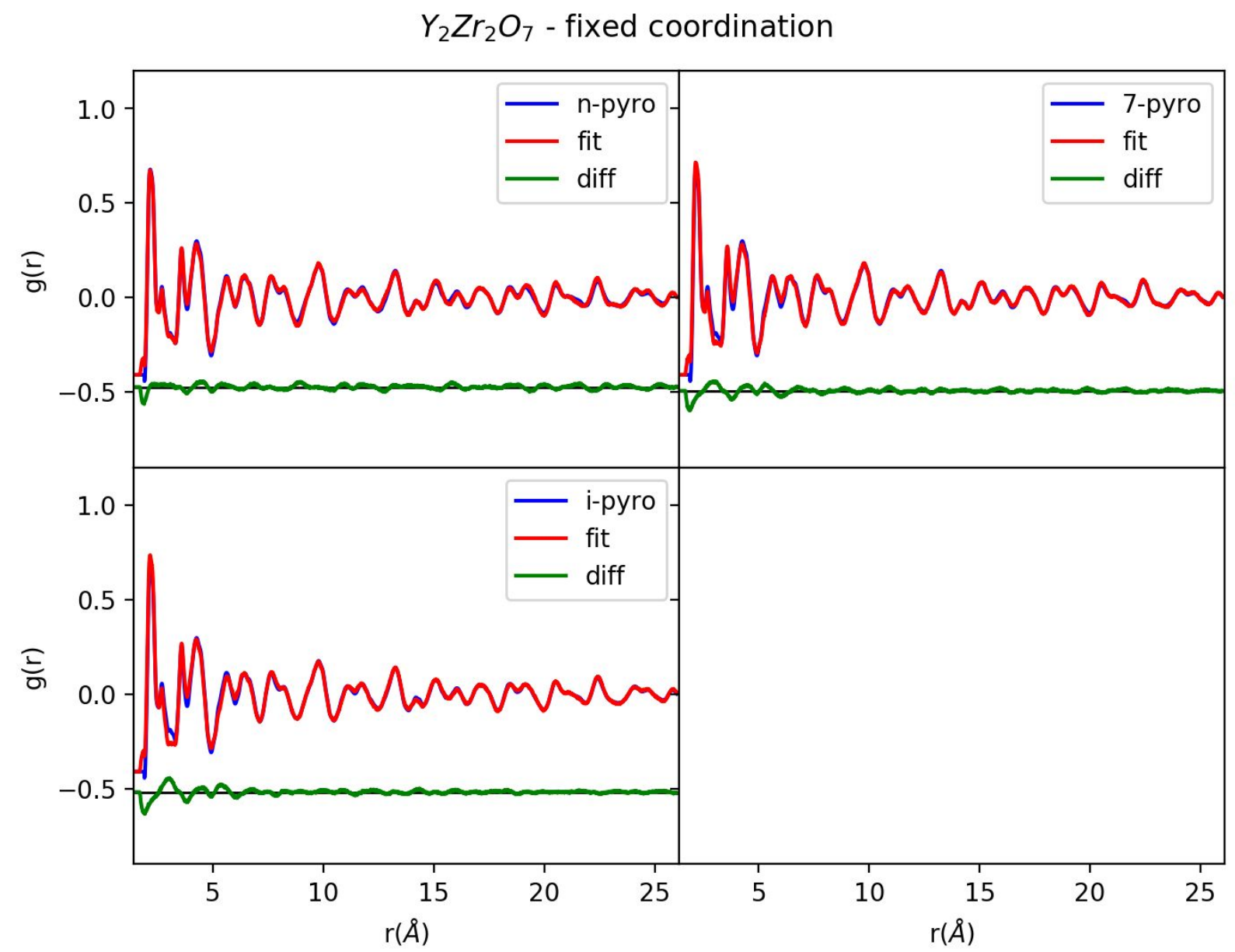

Figure S14: $\mathrm{Y}_{2} \mathrm{Zr}_{2} \mathrm{O}_{7} \mathrm{RMC}$ fits obtained using the 3 different starting configurations and no swapping between vacancies and oxygens, resulting in fixed coordination numbers. 
Refined cation coordinations

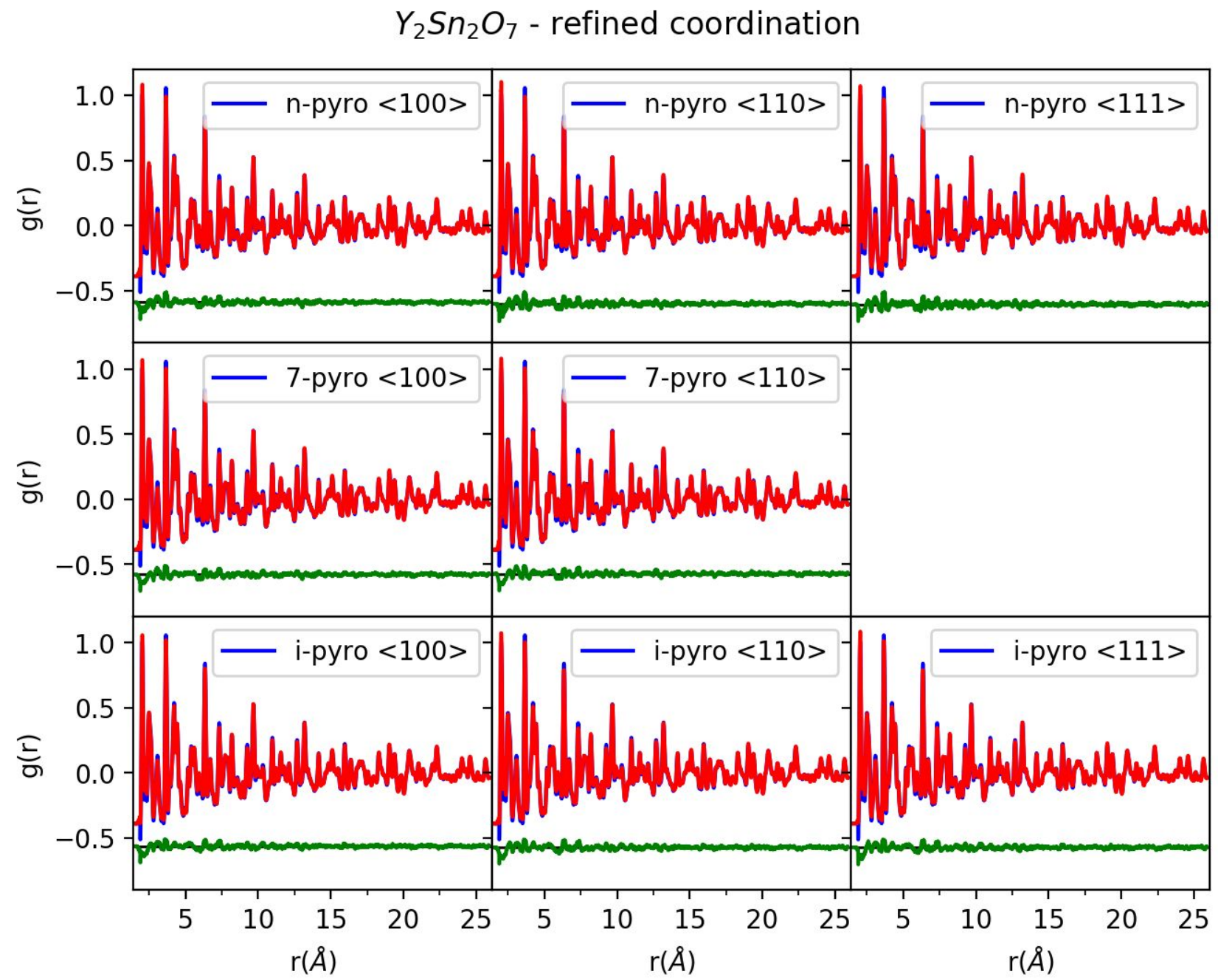

Figure S15: $\mathrm{Y}_{2} \mathrm{Sn}_{2} \mathrm{O}_{7} \mathrm{RMC}$ PDF fits obtained using different refinement strategies based on starting configuration and $V_{O}-V_{O}$ distance constraints, as outlined in the above text. 


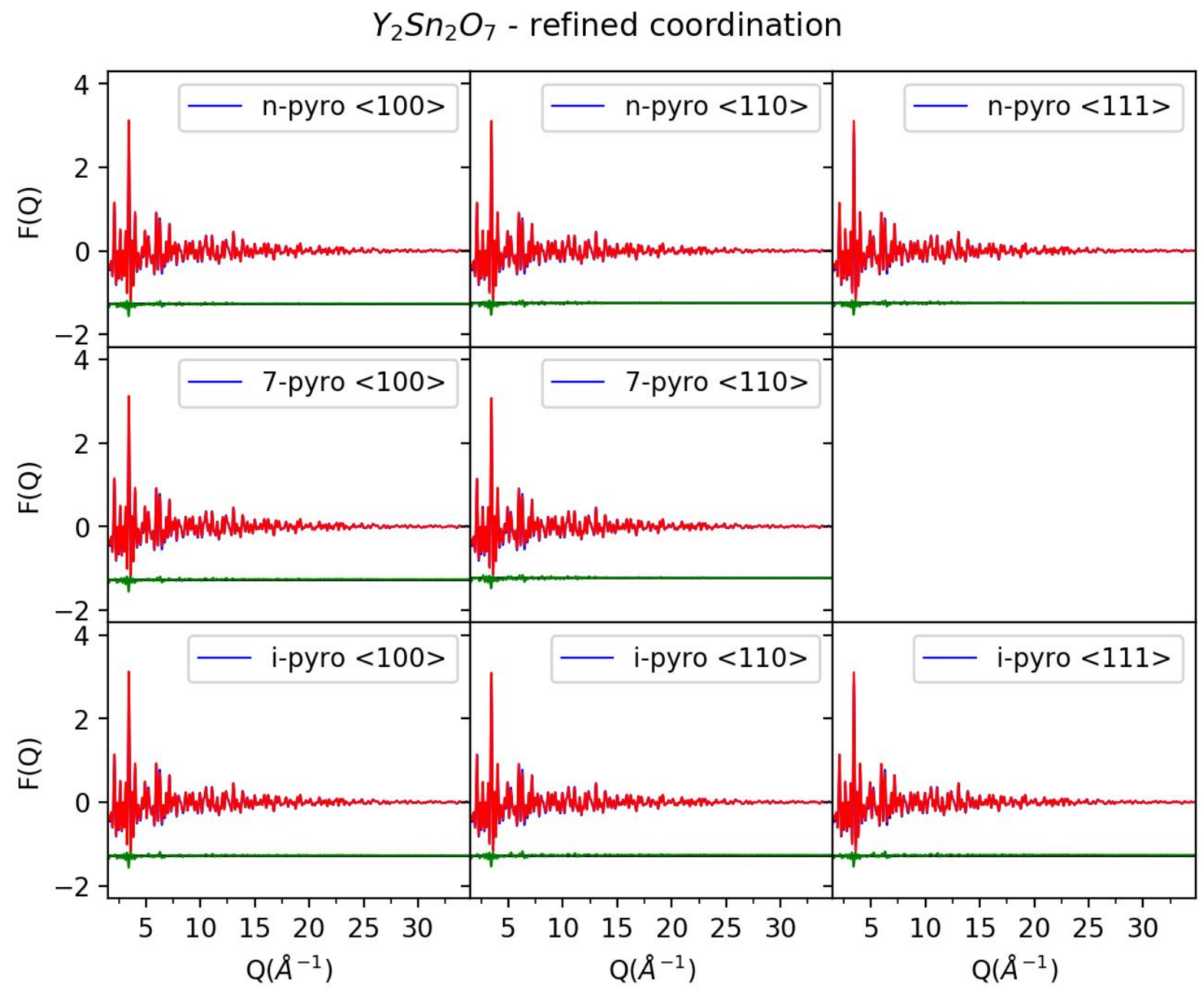

Figure S16: $\mathrm{Y}_{2} \mathrm{Sn}_{2} \mathrm{O}_{7} R M C F(Q)$ fits obtained using different refinement strategies based on starting configuration and $V_{O}-V_{O}$ distance constraints, as outlined in the above text. 


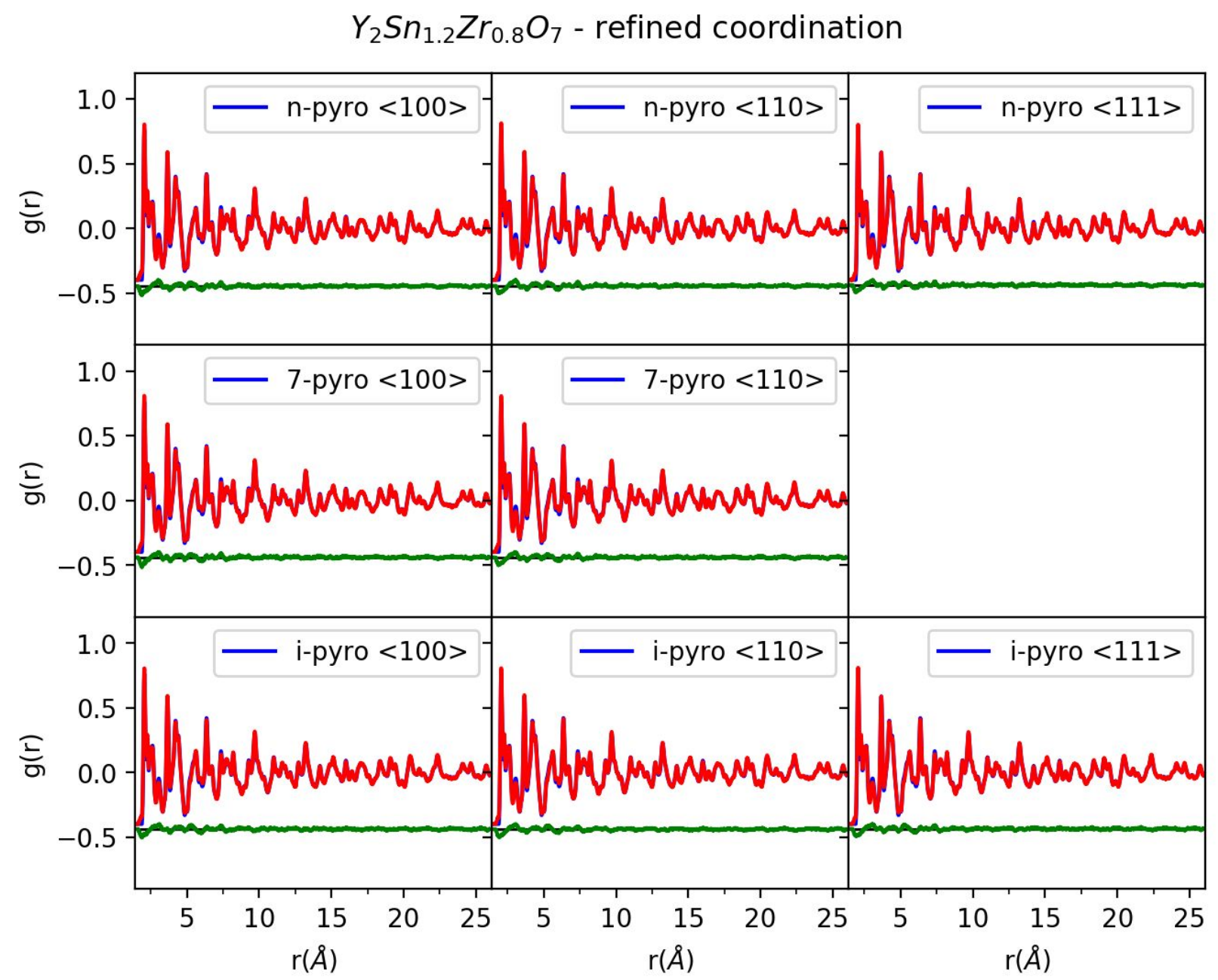

Figure S17: $\mathrm{Y}_{2} \mathrm{Sn}_{1.2} \mathrm{Zr}_{0.8} \mathrm{O}_{7} \mathrm{RMC}$ PDF fits obtained using different refinement strategies based on starting configuration and $V_{O}-V_{O}$ distance constraints, as outlined in the above text. 


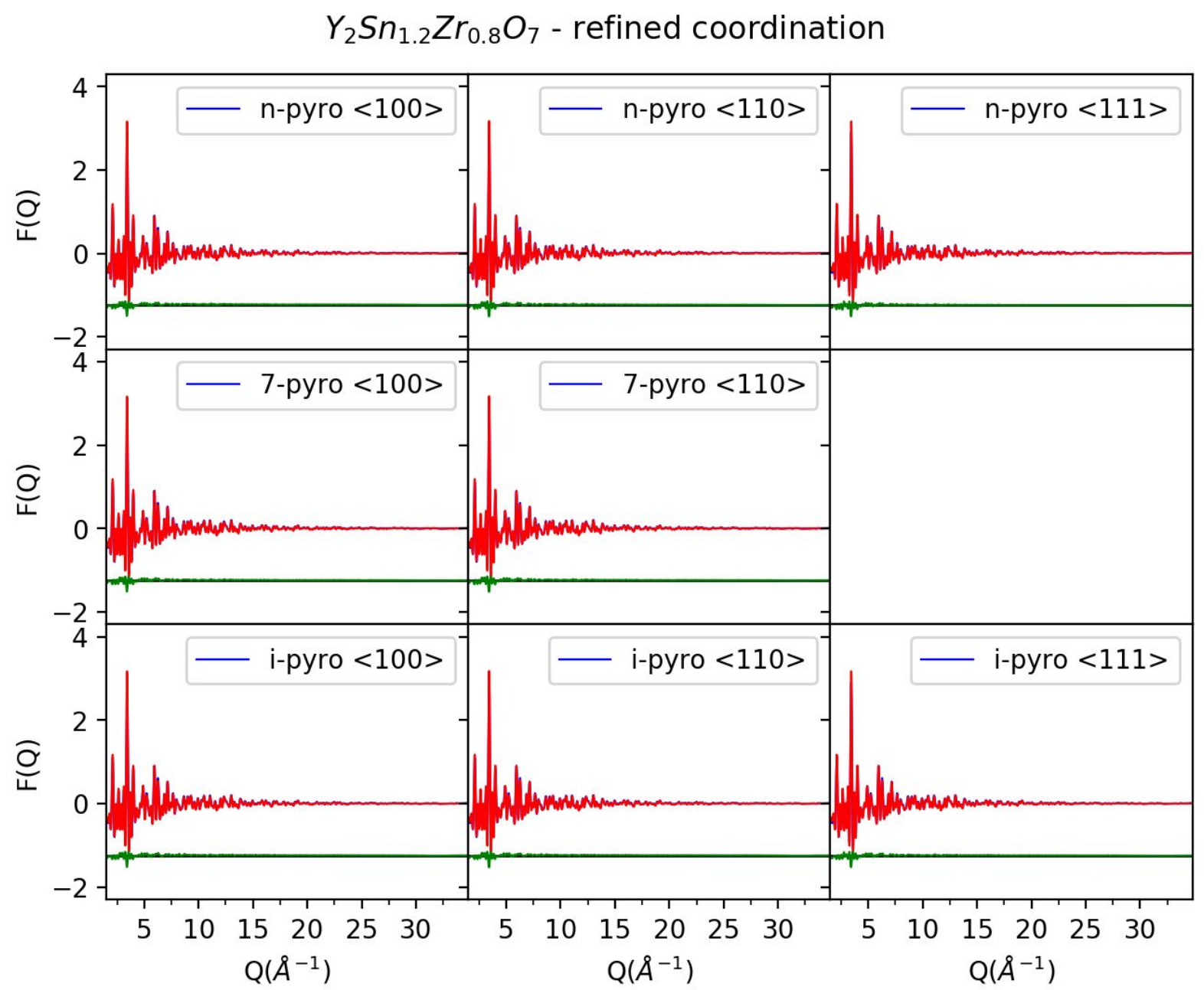

Figure S18: $Y_{2} S_{1.2} Z_{0.8} O_{7} R M C F(Q)$ fits obtained using different refinement strategies based on starting configuration and $V_{O}-V_{O}$ distance constraints, as outlined in the above text. 


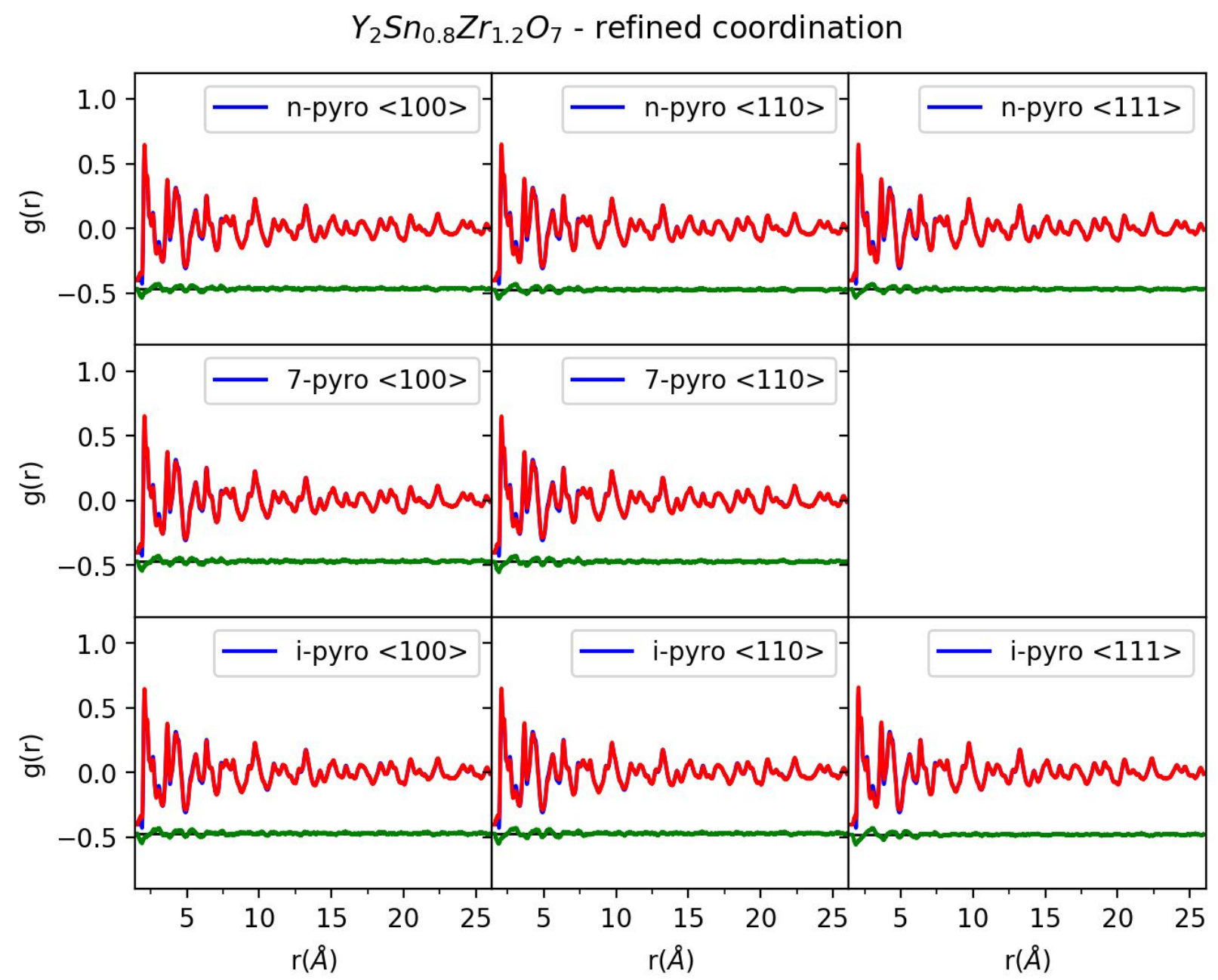

Figure S19: $Y_{2} S_{0.8} Z r_{1.2} O_{7} R M C$ PDF fits obtained using different refinement strategies based on starting configuration and $V_{O}-V_{O}$ distance constraints, as outlined in the above text. 


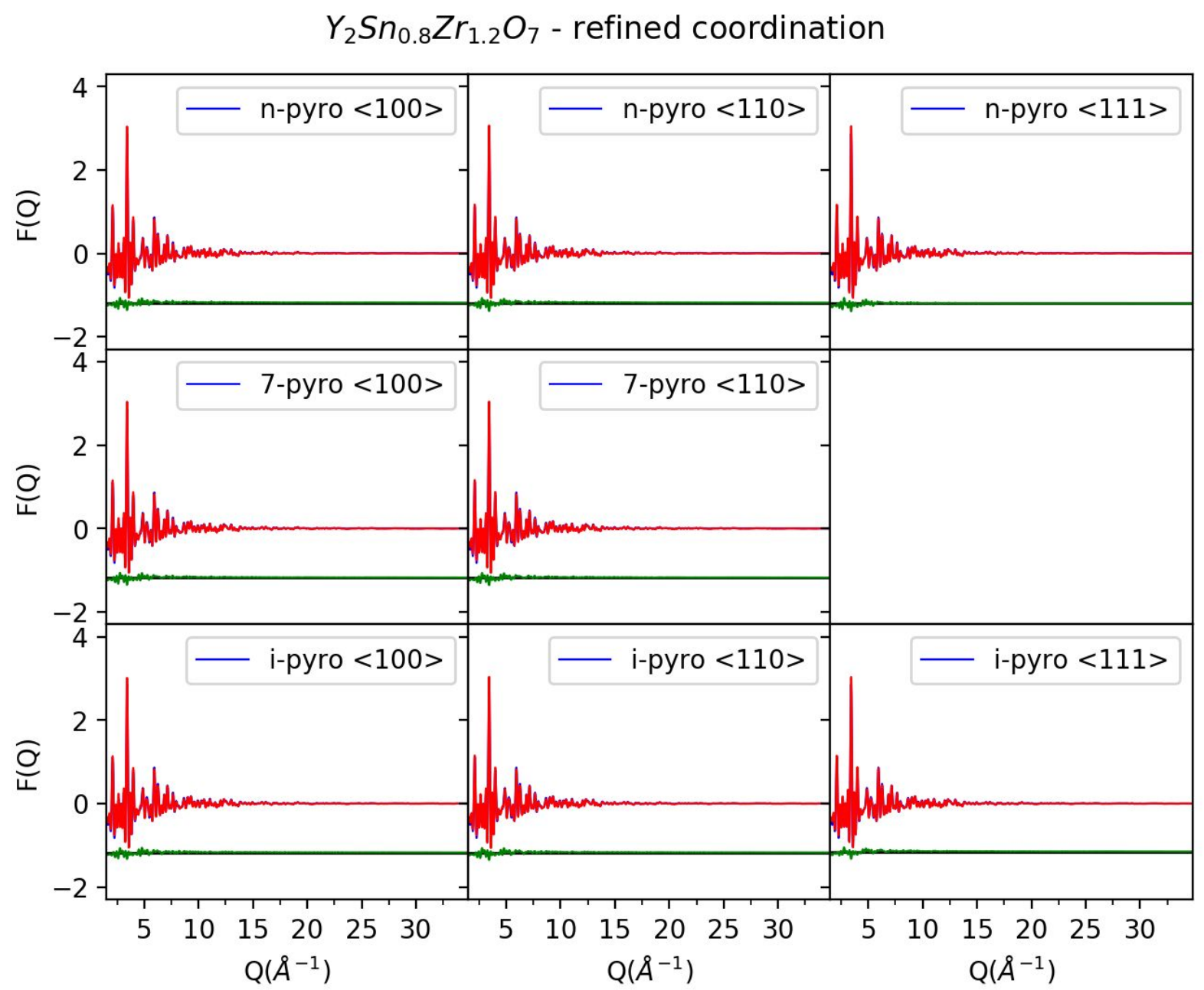

Figure S2O: $\mathrm{Y}_{2} \mathrm{Sn}_{0.8} \mathrm{Zr}_{1.2} \mathrm{O}_{7} R M C F(Q)$ fits obtained using different refinement strategies based on starting configuration and $V_{O}-V_{O}$ distance constraints, as outlined in the above text. 


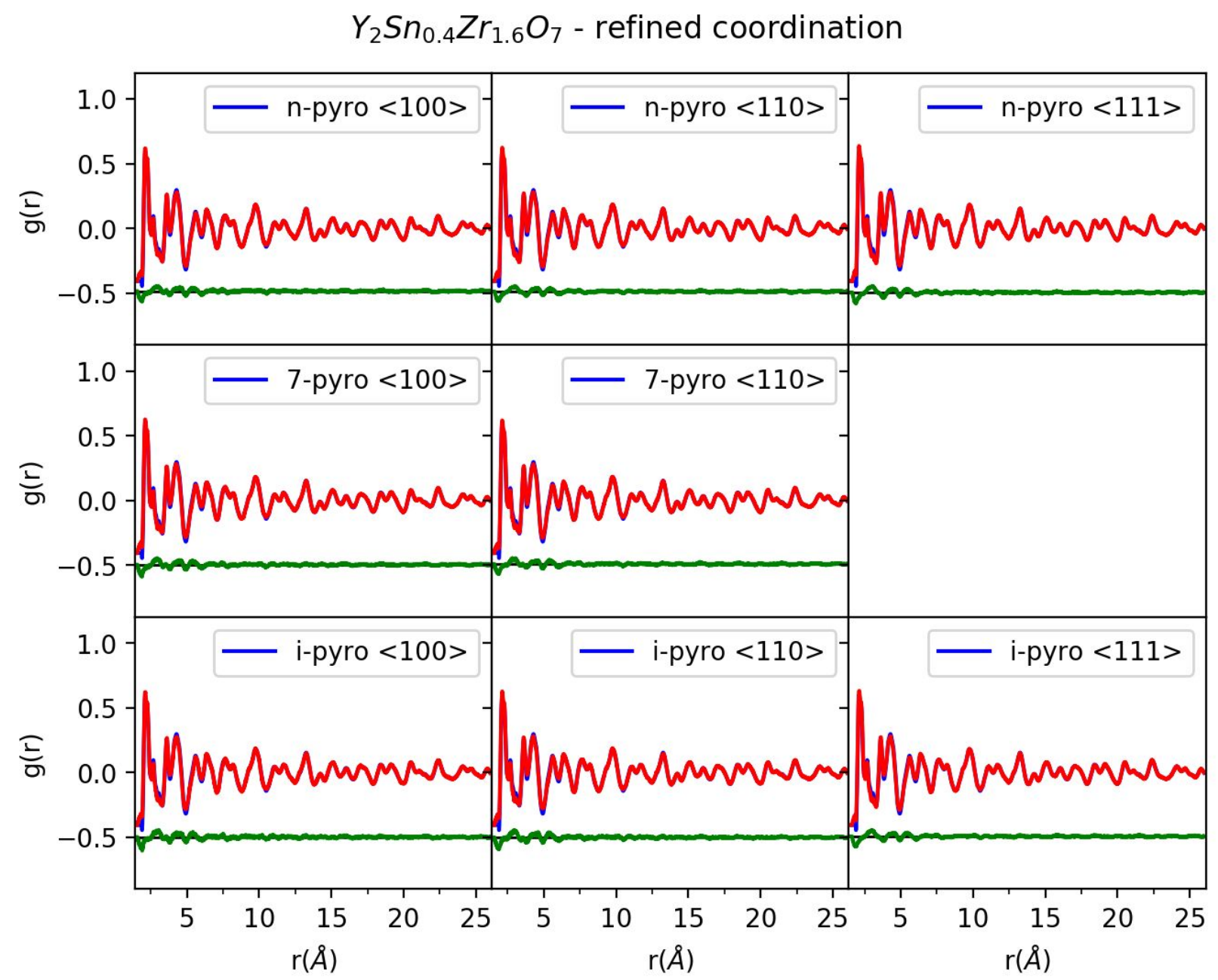

Figure S21: $Y_{2} S_{0.4} Z r_{1.6} O_{7} R M C$ PDF fits obtained using different refinement strategies based on starting configuration and $V_{O}-V_{O}$ distance constraints, as outlined in the above text. 


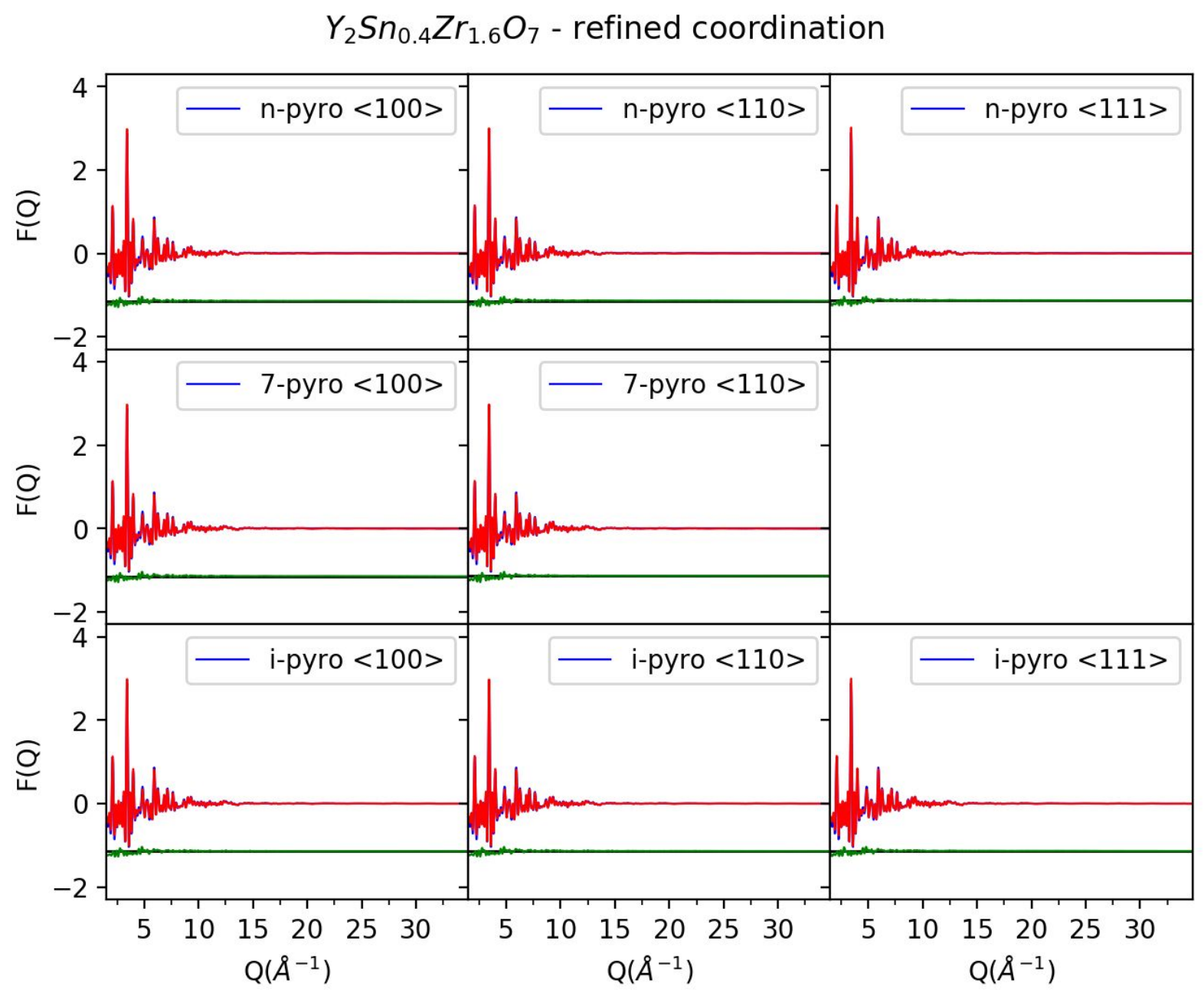

Figure S22: $\mathrm{Y}_{2} \mathrm{Sn}_{0.4} \mathrm{Zr}_{1.6} \mathrm{O}_{7} R M C F(Q)$ fits obtained using different refinement strategies based on starting configuration and $V_{O}-V_{O}$ distance constraints, as outlined in the above text. 


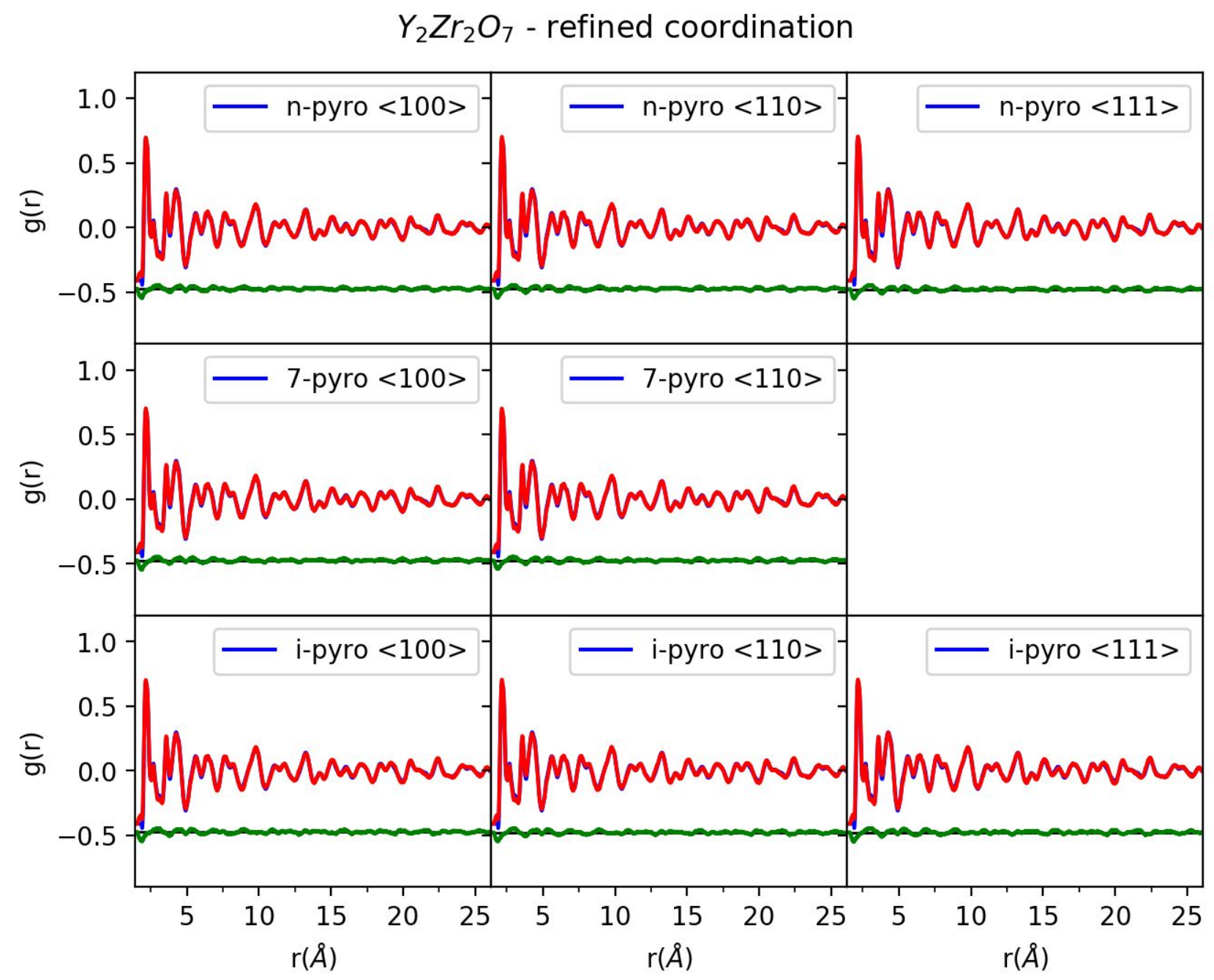

Figure S23: $\mathrm{Y}_{2} \mathrm{Zr}_{2} \mathrm{O}_{7}$ RMC PDF fits obtained using different refinement strategies based on starting configuration and $V_{O^{-}} V_{O}$ distance constraints, as outlined in the above text. 


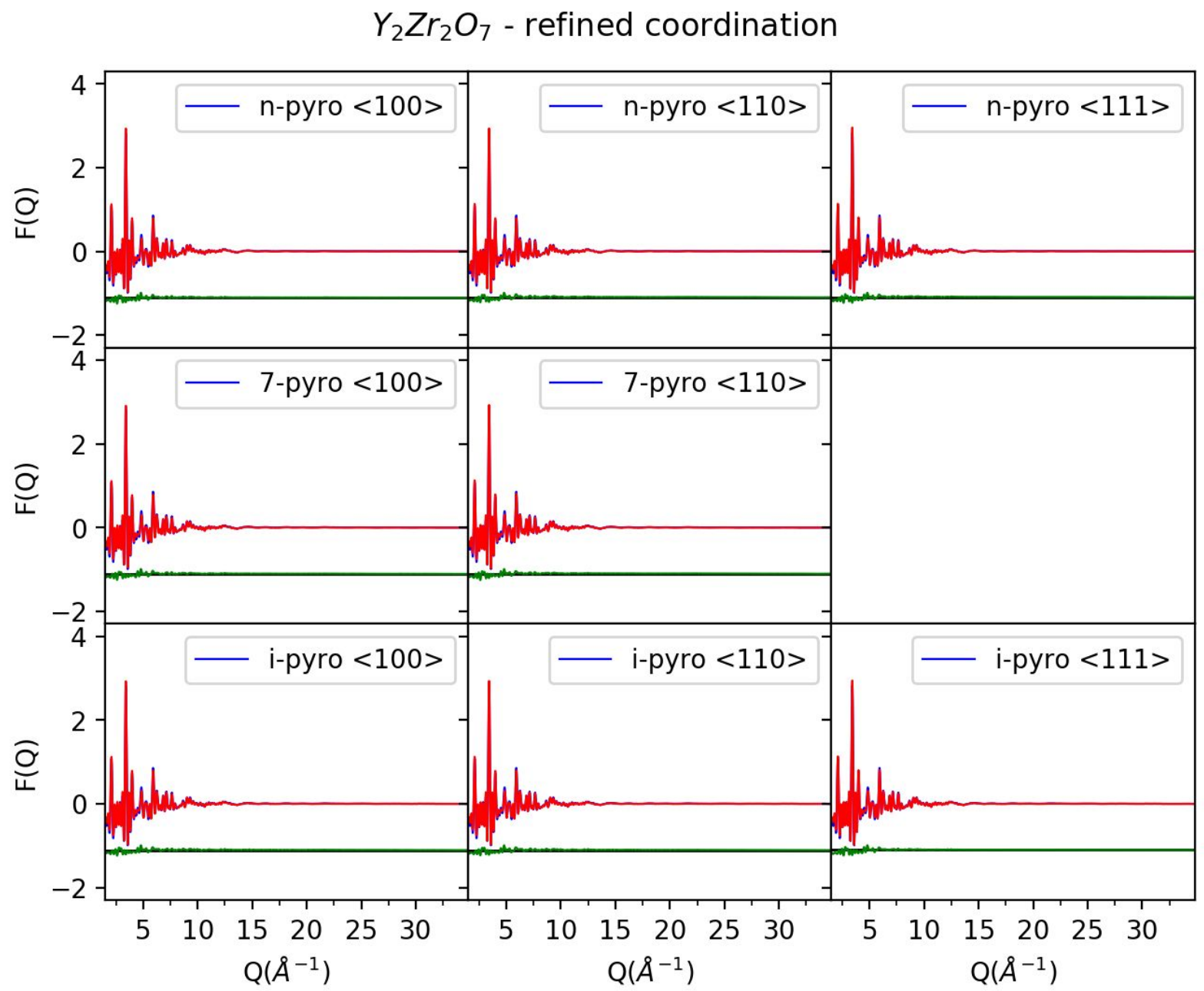

Figure S24: $\mathrm{Y}_{2} \mathrm{Zr}_{2} \mathrm{O}_{7} R M C F(Q)$ fits obtained using different refinement strategies based on starting configuration and $V_{O}-V_{O}$ distance constraints, as outlined in the above text. 


\section{Parials}

Figure S25 and Figure S26 show the partial PDFs of the relevant pairs for all refinement strategies.

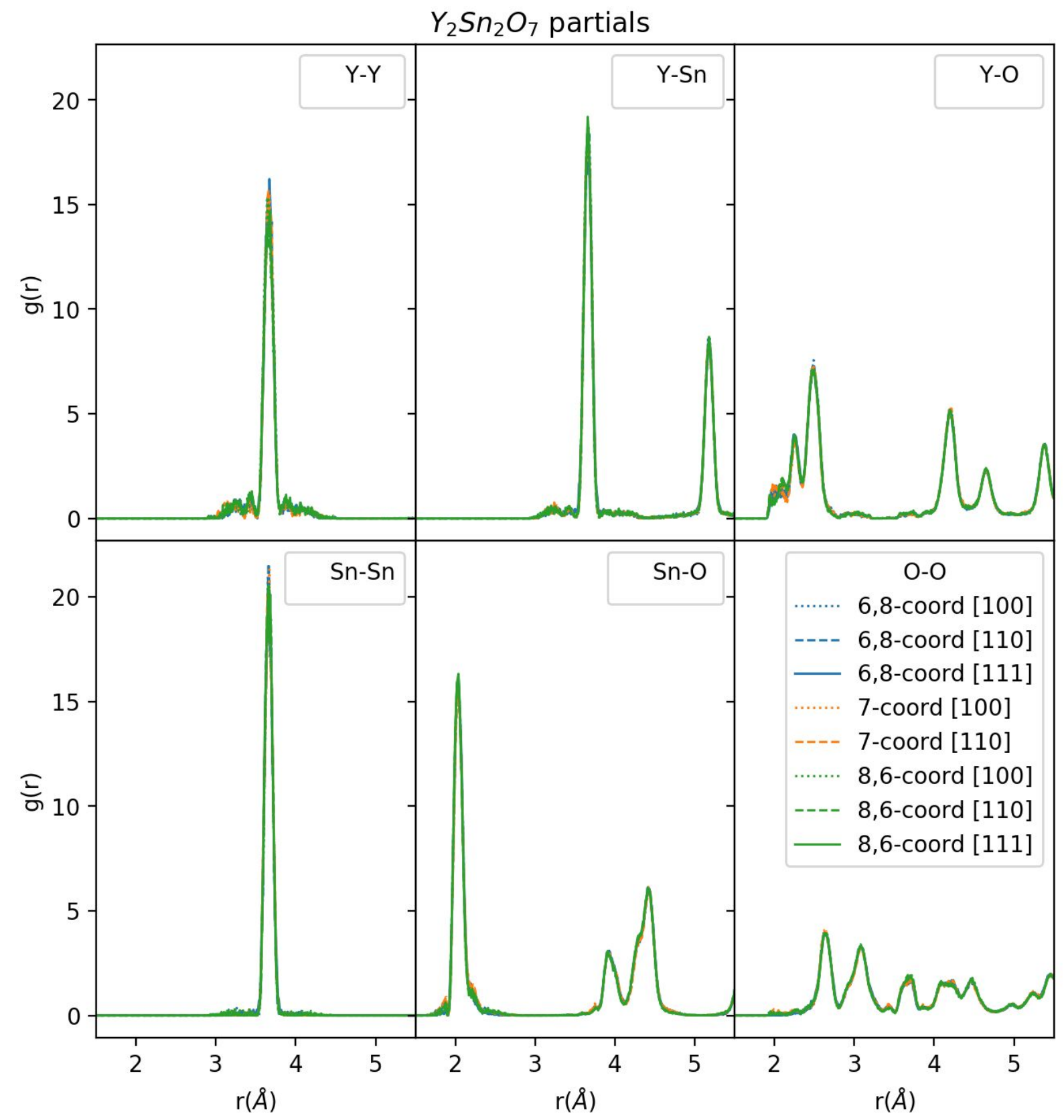

Figure S25: $\mathrm{Y}_{2} \mathrm{Sn}_{2} \mathrm{O}_{7}$ partial PDFs from RMC fits obtained using different refinement strategies based on starting configuration and $V_{O}-V_{O}$ distance constraints, as outlined in the above text. 


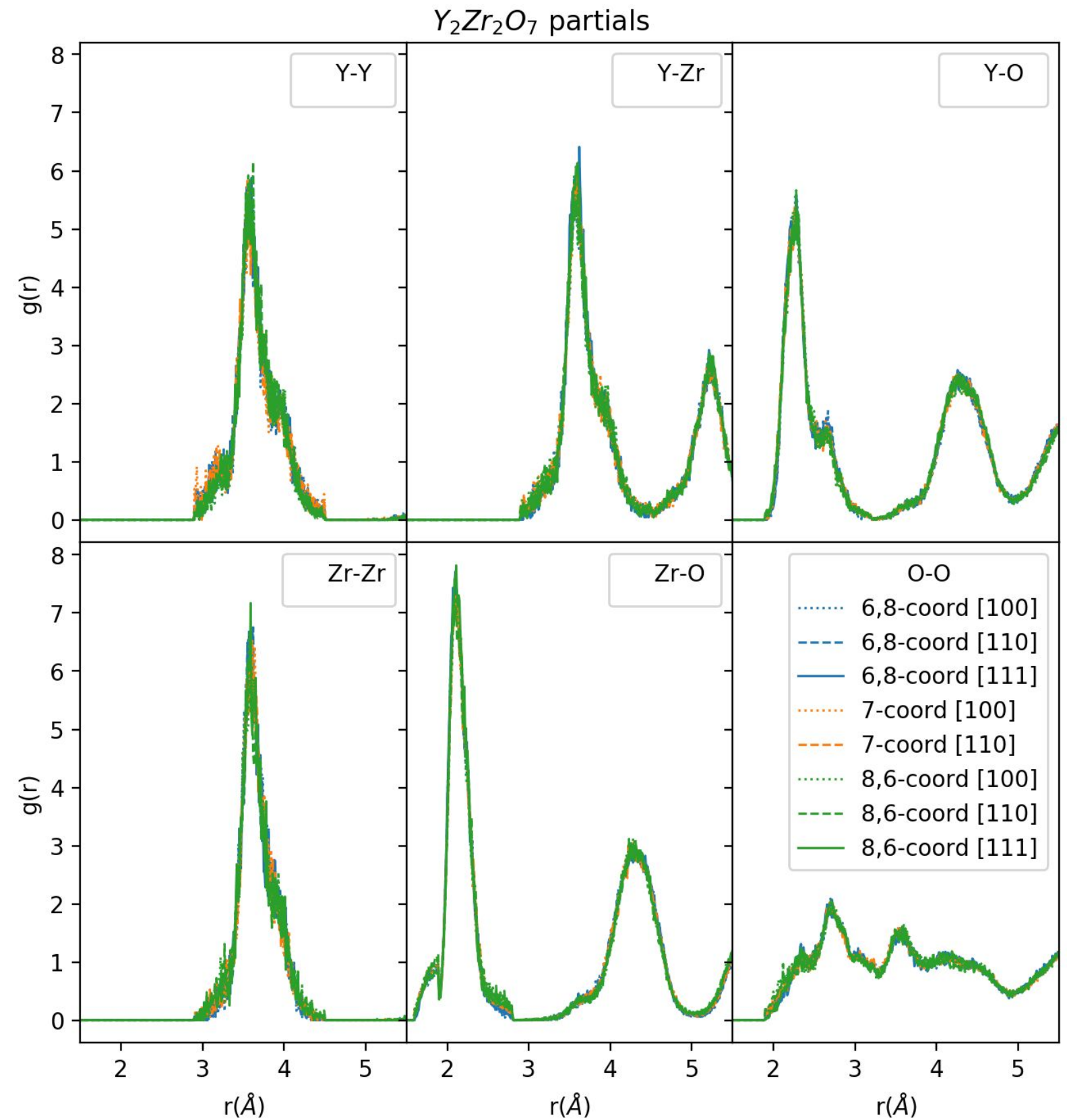

Figure S26: $\mathrm{Y}_{2} \mathrm{Zr}_{2} \mathrm{O}_{7}$ partial PDFs from RMC fits obtained using different refinement strategies based on starting configuration and $V_{O}-V_{O}$ distance constraints, as outlined in the above text. 


\section{Displacements}

\section{Strategy overlay}

Figure S27 and Figure S28 show the histograms of atomic displacements along the $x, y$ and $z$ directions. In this case, atoms and $x, y$ and $z$ have been plotted separately, with refinement strategy plotted on top of each other. 


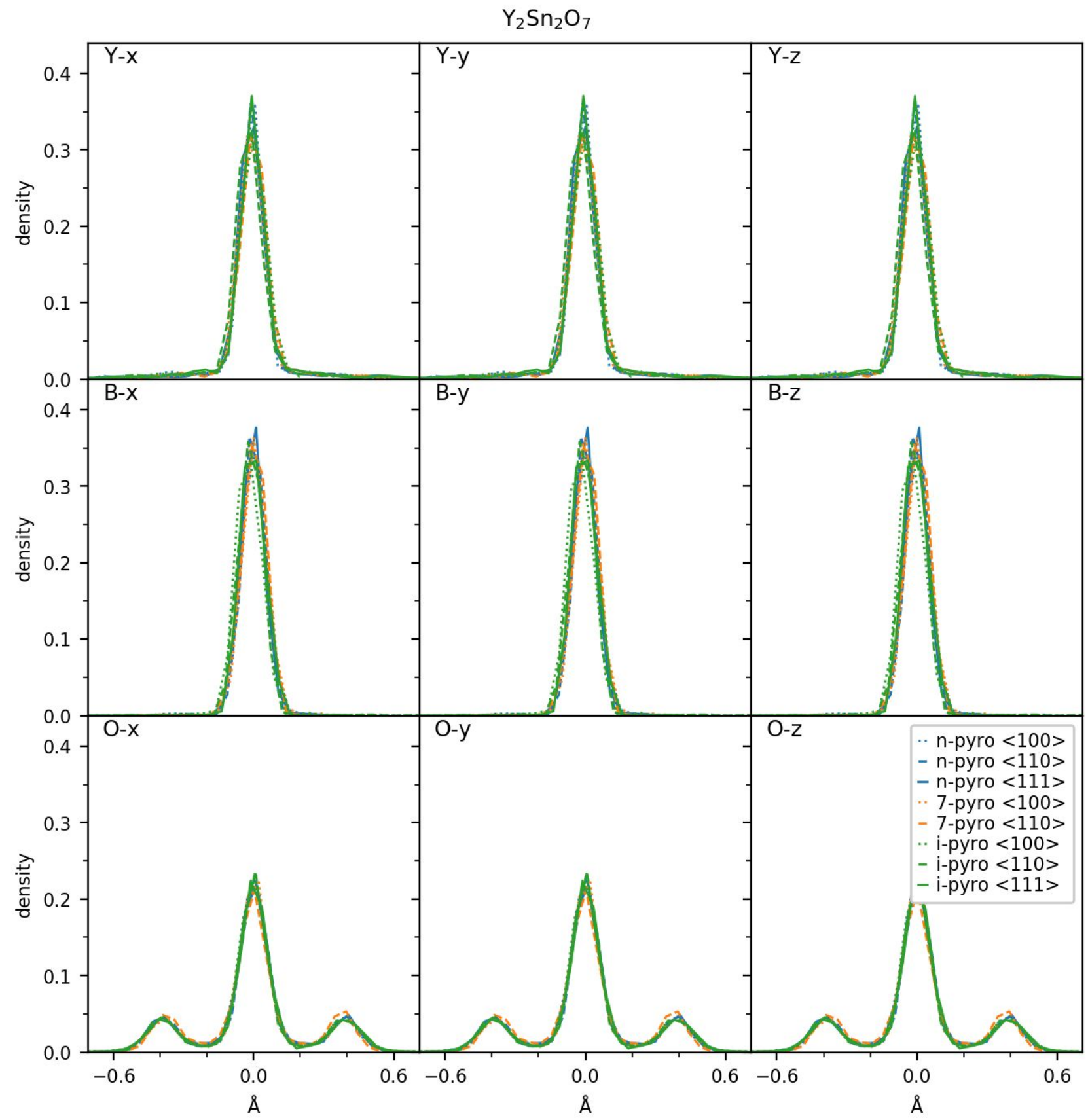

Figure S27: $\mathrm{Y}_{2} \mathrm{Sn}_{2} \mathrm{O}_{7}$ atomic displacement distributions from RMC fits obtained using different refinement strategies based on starting configuration and $V_{O}-V_{O}$ distance constraints, as outlined in the above text. In this figure, the distributions have been seperated by atom $(A, B$ and $O)$ and axis ( $x, y$ and $z)$, but overlaid by refinement strategy. 


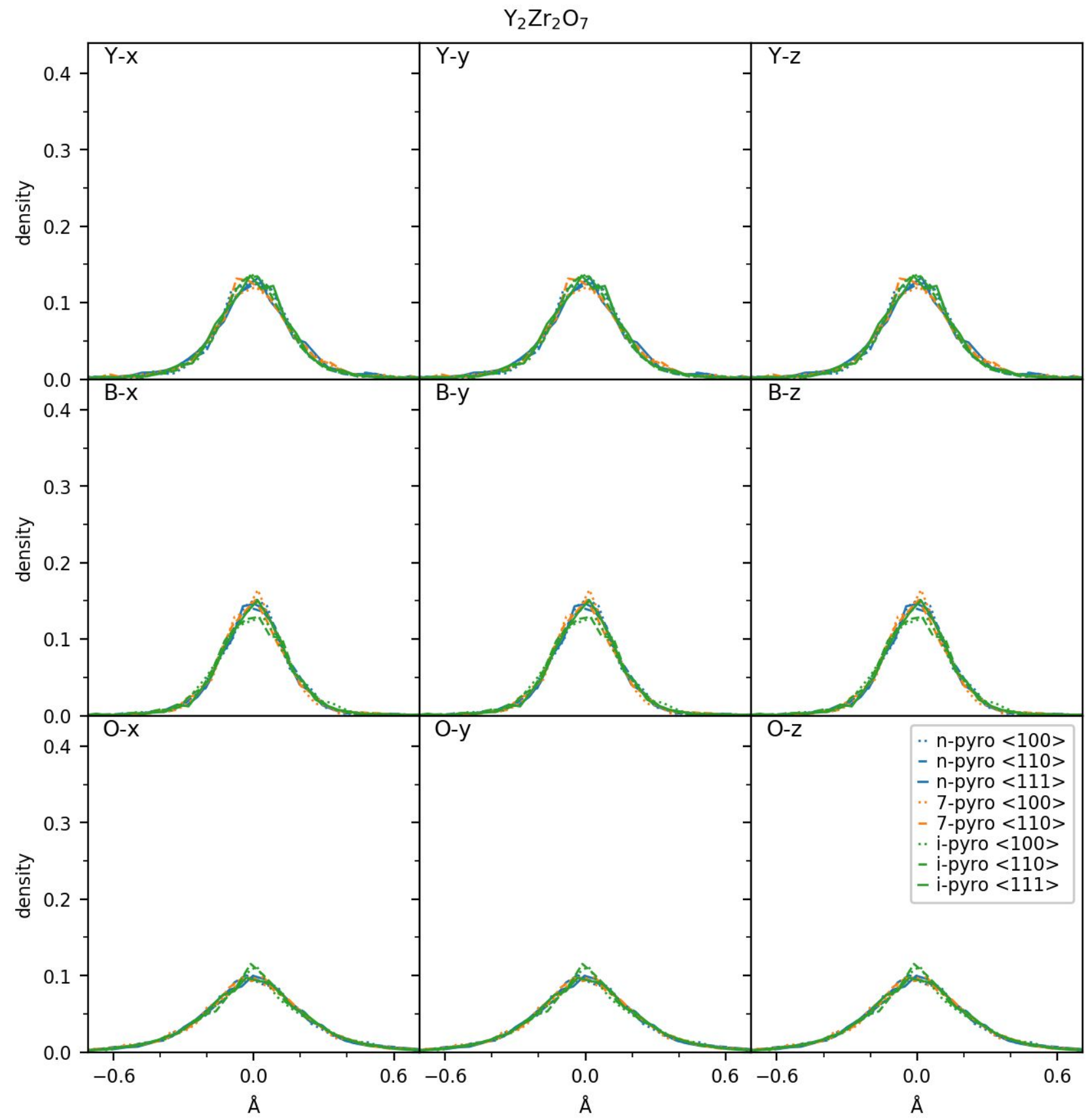

Figure S28: $\mathrm{Y}_{2} \mathrm{Zr}_{2} \mathrm{O}_{7}$ atomic displacement distributions from RMC fits obtained using different refinement strategies based on starting configuration and $V_{O}-V_{O}$ distance constraints, as outlined in the above text. In this figure, the distributions have been seperated by atom $(A, B$ and $O)$ and axis ( $x, y$ and $z)$, but overlaid by refinement strategy. 


\section{Xyz overlay}

Figure S29 - Figure S34 show the histograms of atomic displacements along the $x, y$ and $z$ directions. In this case, refinement strategy and atoms have been plotted separately, with $x, y$ and $z$ plotted on top of each other.

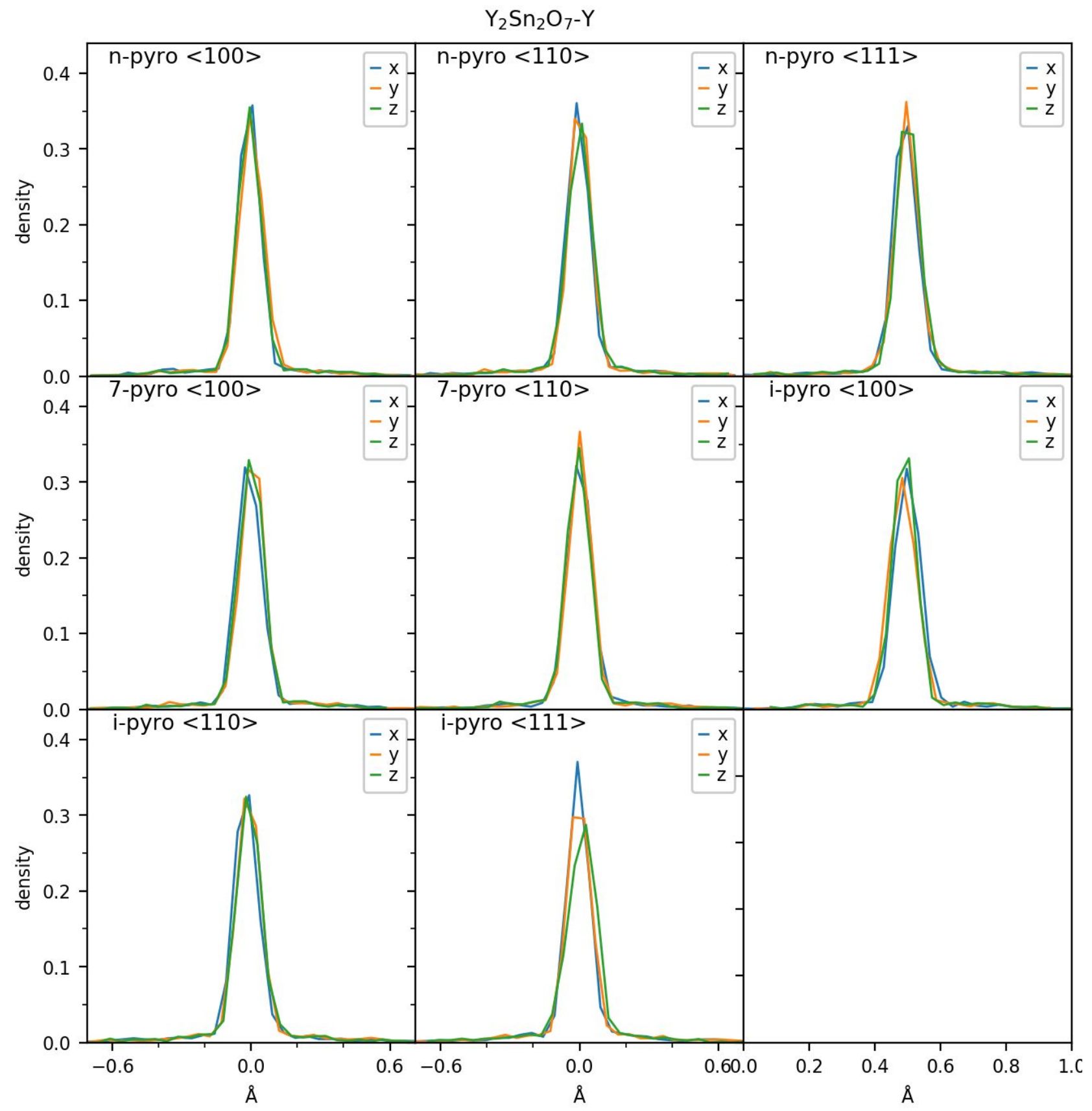

Figure S29: $\mathrm{Y}_{2} \mathrm{Sn}_{2} \mathrm{O}_{7}$ atomic displacement distributions of $Y$ atoms from RMC fits obtained using different refinement strategies based on starting configuration and $V_{O}-V_{O}$ distance constraints, as outlined in the above text. In this figure, the distributions have been seperated by refinement strategy, but overlaid by $x, y$ and $z$. 


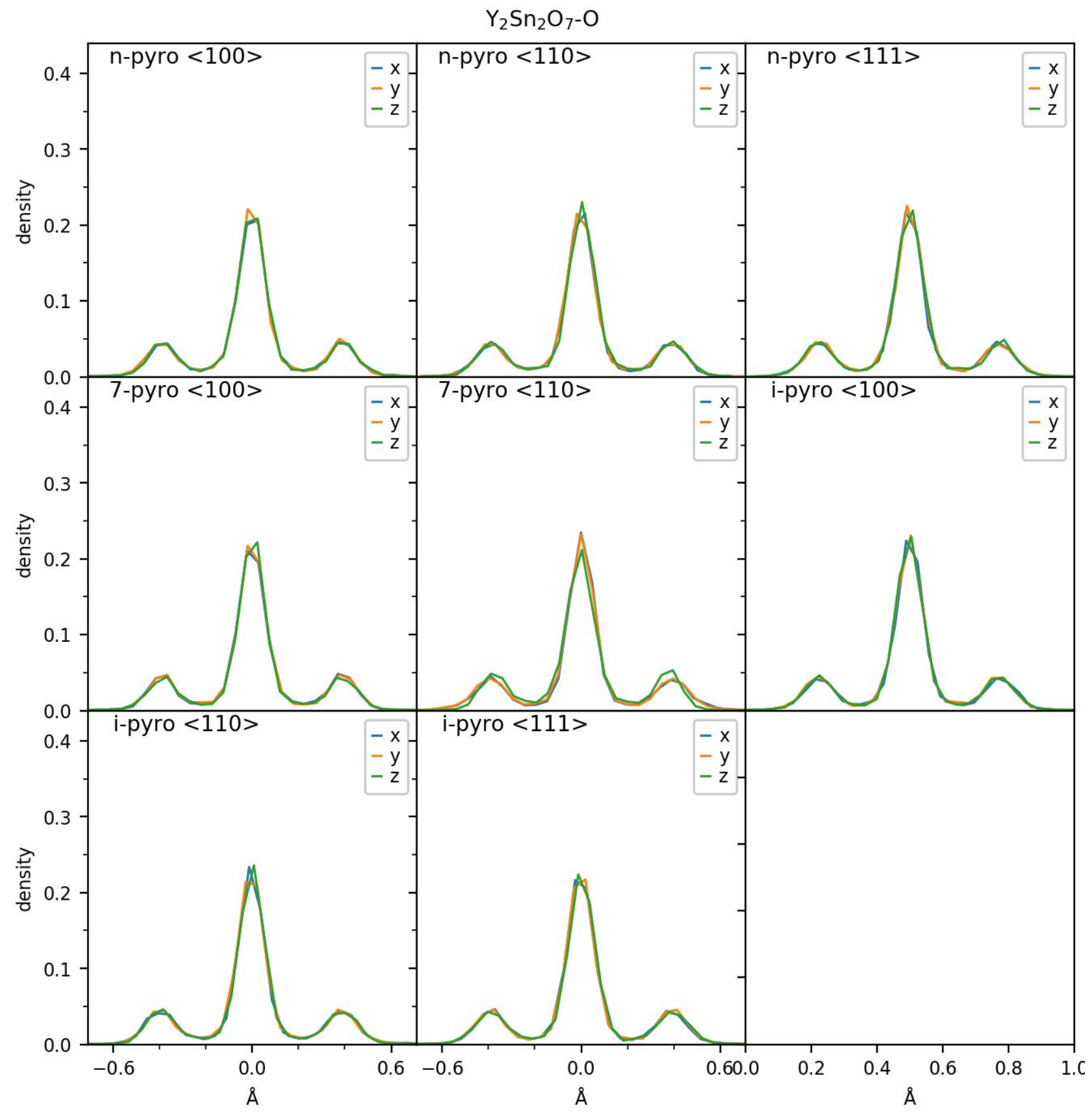

Figure S30: $\mathrm{Y}_{2} \mathrm{Sn}_{2} \mathrm{O}_{7}$ atomic displacement distributions of $\mathrm{O}$ atoms from $\mathrm{RMC}$ fits obtained using different refinement strategies based on starting configuration and $V_{O}-V_{O}$ distance constraints, as outlined in the above text. In this figure, the distributions have been seperated by refinement strategy, but overlaid by $x, y$ and $z$. 


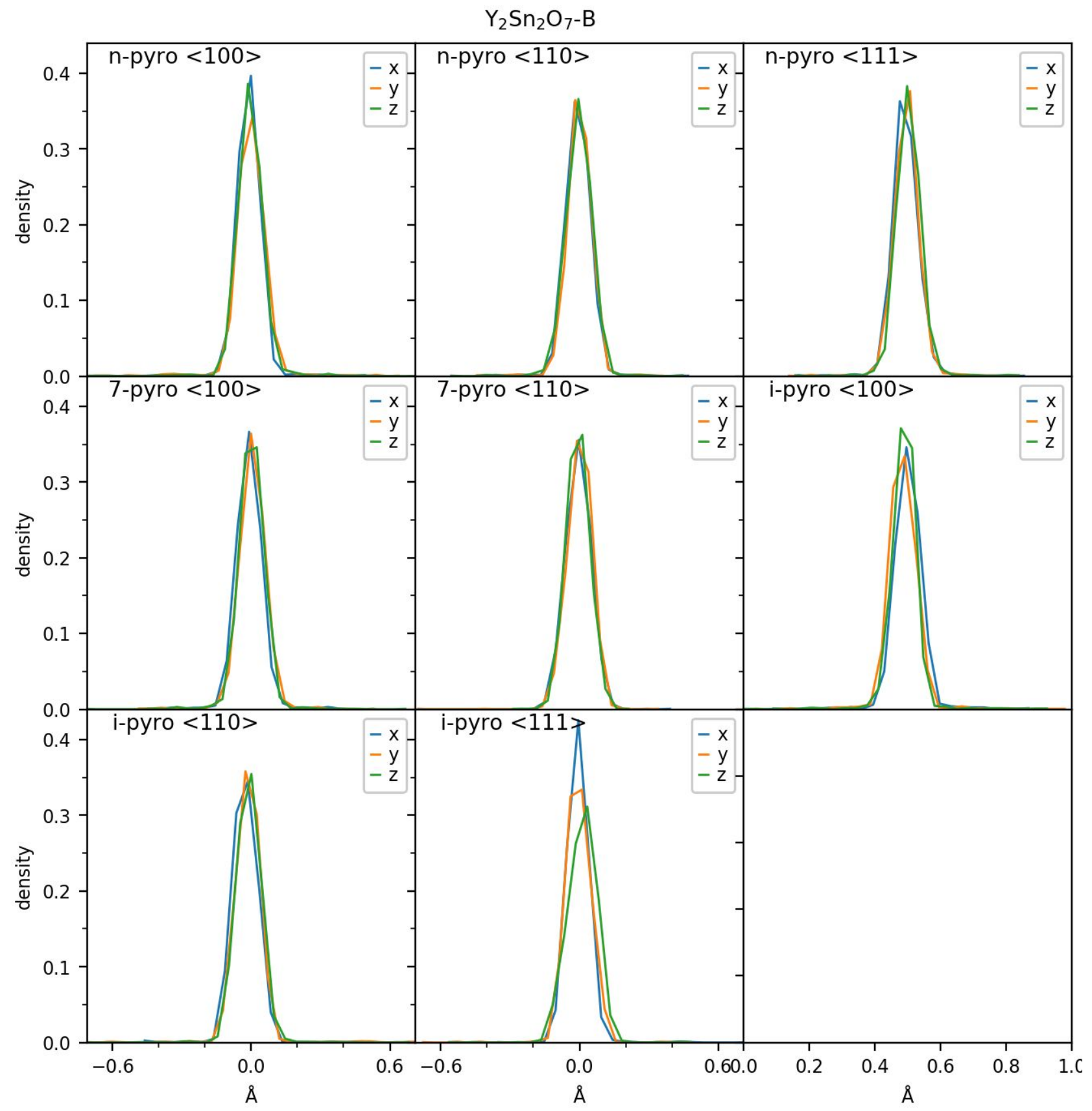

Figure S31: $\mathrm{Y}_{2} \mathrm{Sn}_{2} \mathrm{O}_{7}$ atomic displacement distributions of $B(\mathrm{Sn})$ atoms from RMC fits obtained using different refinement strategies based on starting configuration and $V_{O}-V_{O}$ distance constraints, as outlined in the above text. In this figure, the distributions have been seperated by refinement strategy, but overlaid by $x, y$ and $z$. 


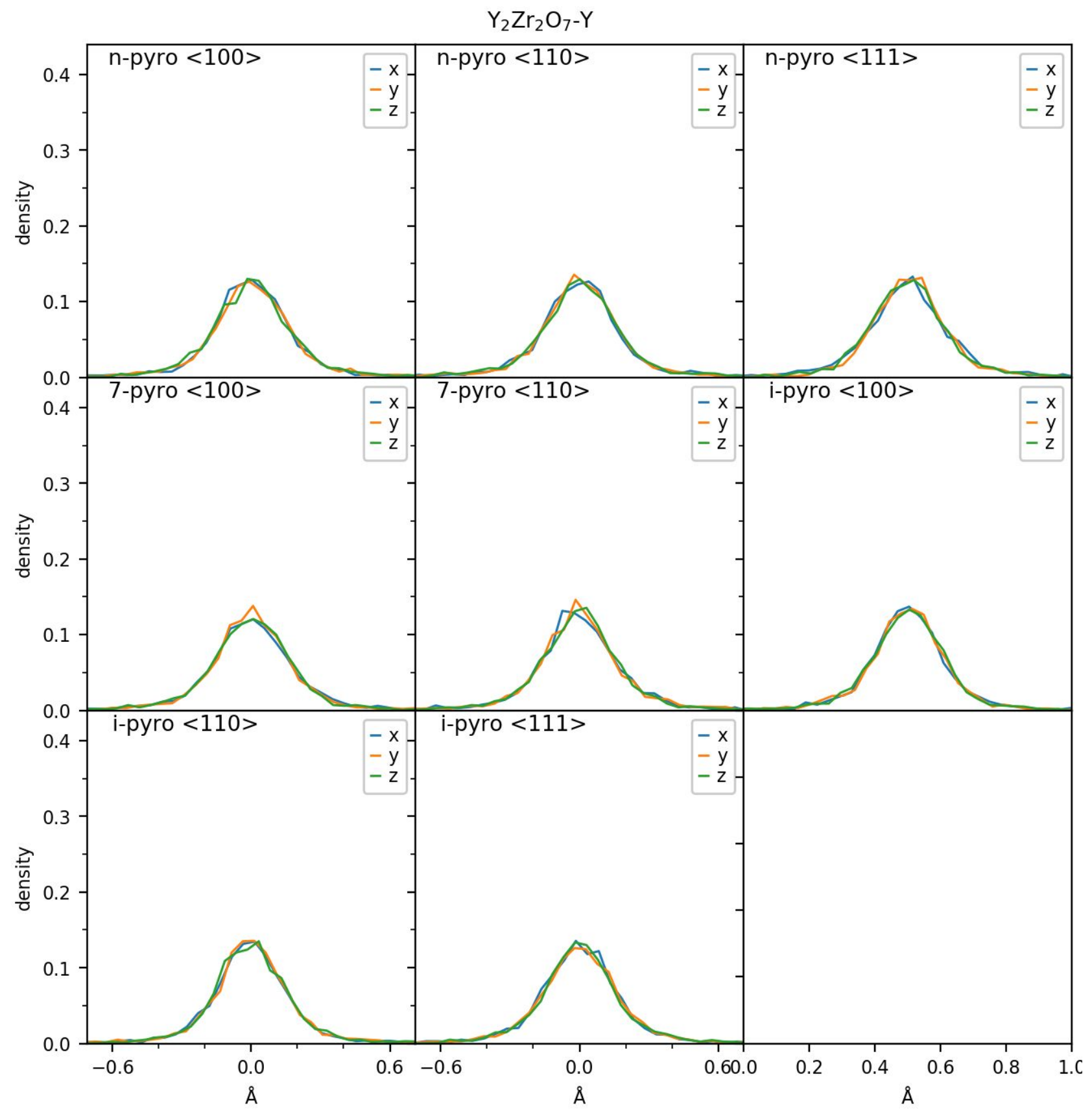

Figure S32: $\mathrm{Y}_{2} \mathrm{Zr}_{2} \mathrm{O}_{7}$ atomic displacement distributions of $\mathrm{Y}$ atoms from RMC fits obtained using different refinement strategies based on starting configuration and $V_{O}-V_{O}$ distance constraints, as outlined in the above text. In this figure, the distributions have been seperated by refinement strategy, but overlaid by $x, y$ and $z$. 


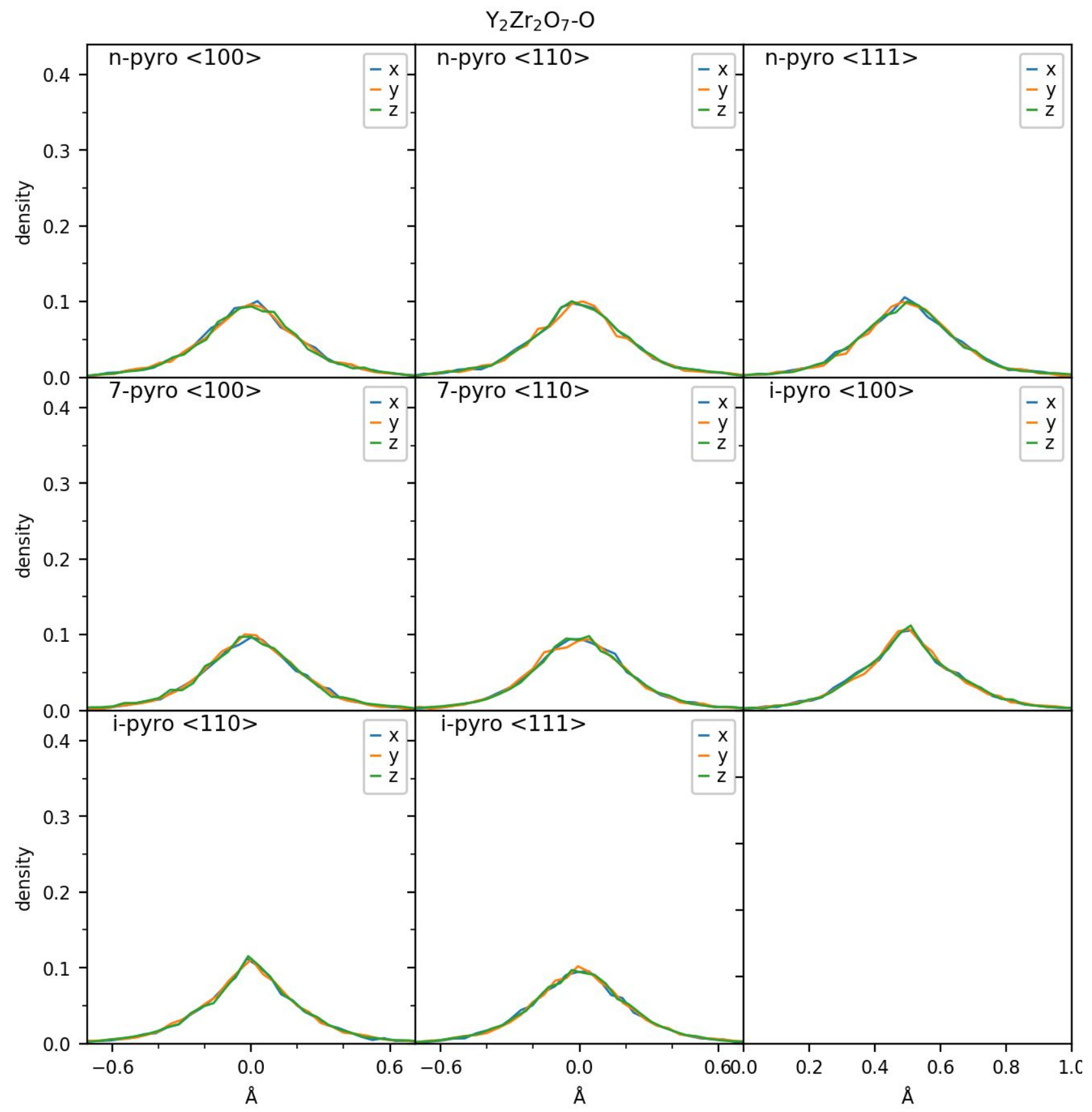

Figure S33: $\mathrm{Y}_{2} \mathrm{Zr}_{2} \mathrm{O}_{7}$ atomic displacement distributions of $\mathrm{O}$ atoms from $\mathrm{RMC}$ fits obtained using different refinement strategies based on starting configuration and $V_{O}-V_{O}$ distance constraints, as outlined in the above text. In this figure, the distributions have been seperated by refinement strategy, but overlaid by $x, y$ and $z$. 


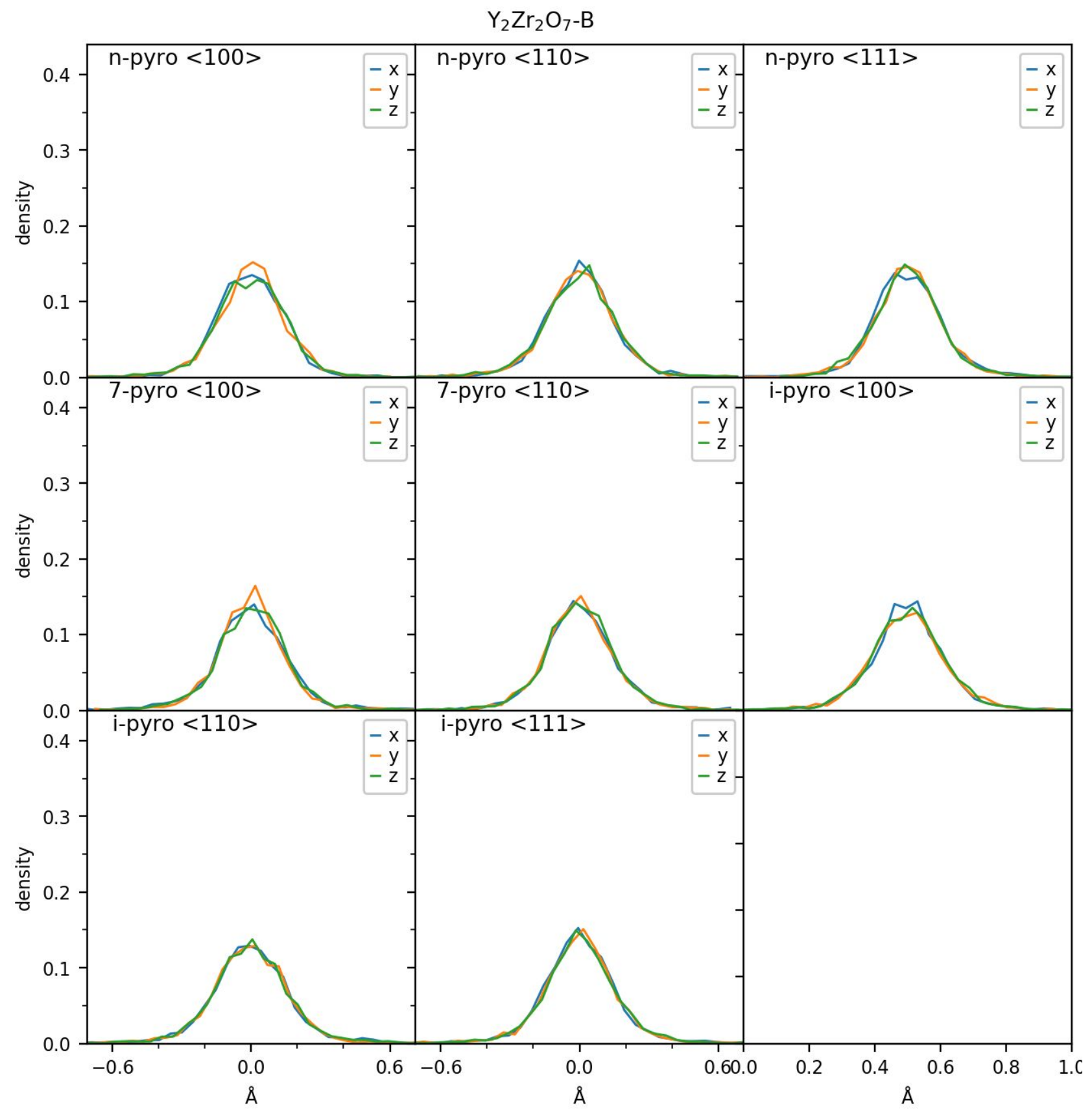

Figure S34: $\mathrm{Y}_{2} \mathrm{Zr}_{2} \mathrm{O}_{7}$ atomic displacement distributions of $B(\mathrm{Zr})$ atoms from $\mathrm{RMC}$ fits obtained using different refinement strategies based on starting configuration and $V_{O}-V_{O}$ distance constraints, as outlined in the above text. In this figure, the distributions have been seperated by refinement strategy, but overlaid by $x, y$ and $z$. 


\section{Bond angles}

Figure S35 and Figure S36 show the histograms of the bond angles for the A-site, B-site, O-site and Msites (labelled M-M-M angles), where $\mathrm{M}$ refers to all cations.

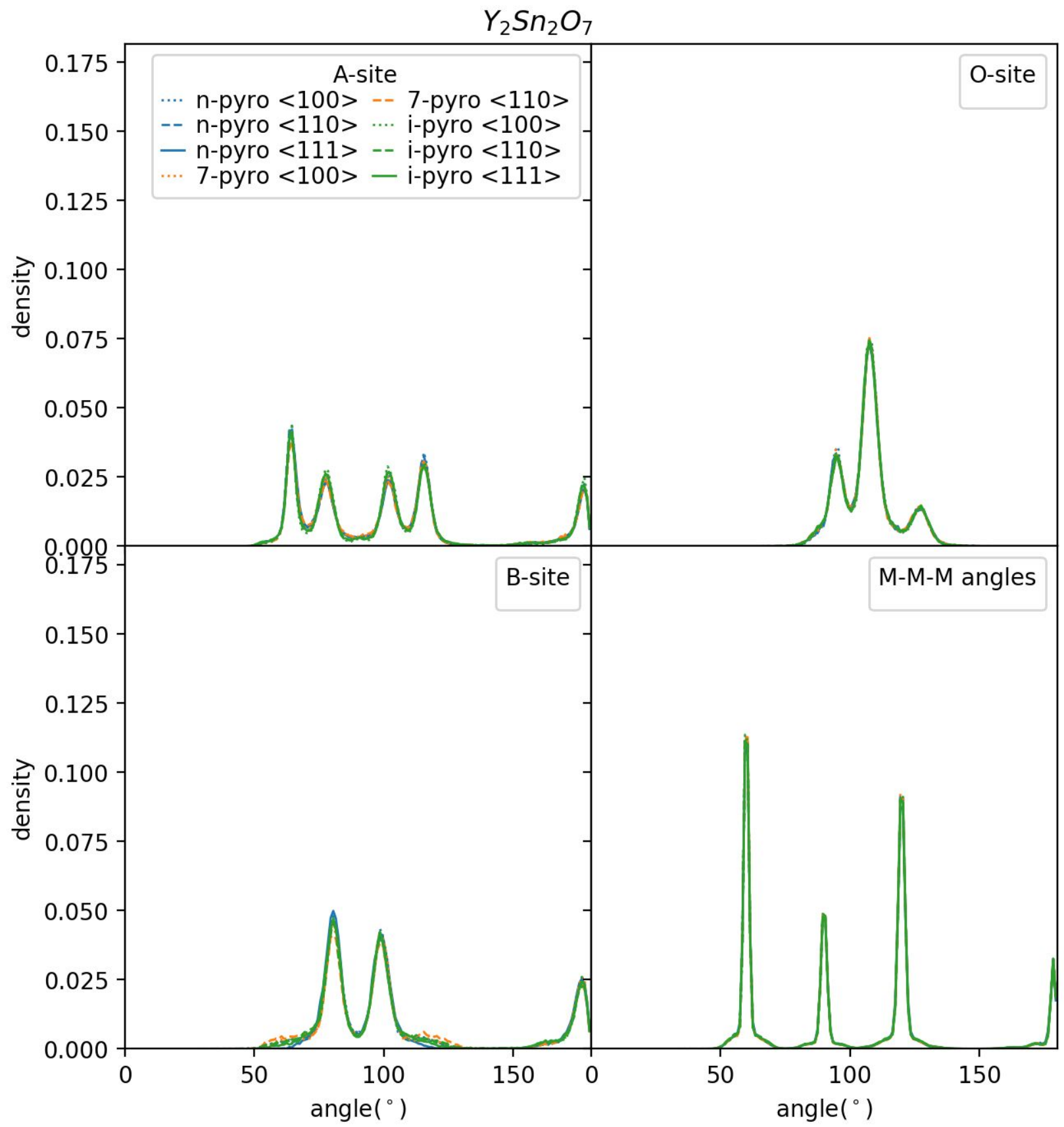

Figure S35: $\mathrm{Y}_{2} \mathrm{Sn}_{2} \mathrm{O}_{7}$ inter-atomic angles extracted from the RMC refinements. The A-site refers to $O-A-O$ angles, the $B$-site refers to $O-B-O$ angles, the $O$-site refers to $O-M-O$ angles and $M-M-M$ angles refers to all angles between nearest-neighbor cations. The distributions have been overlaid by refinement strategy. 


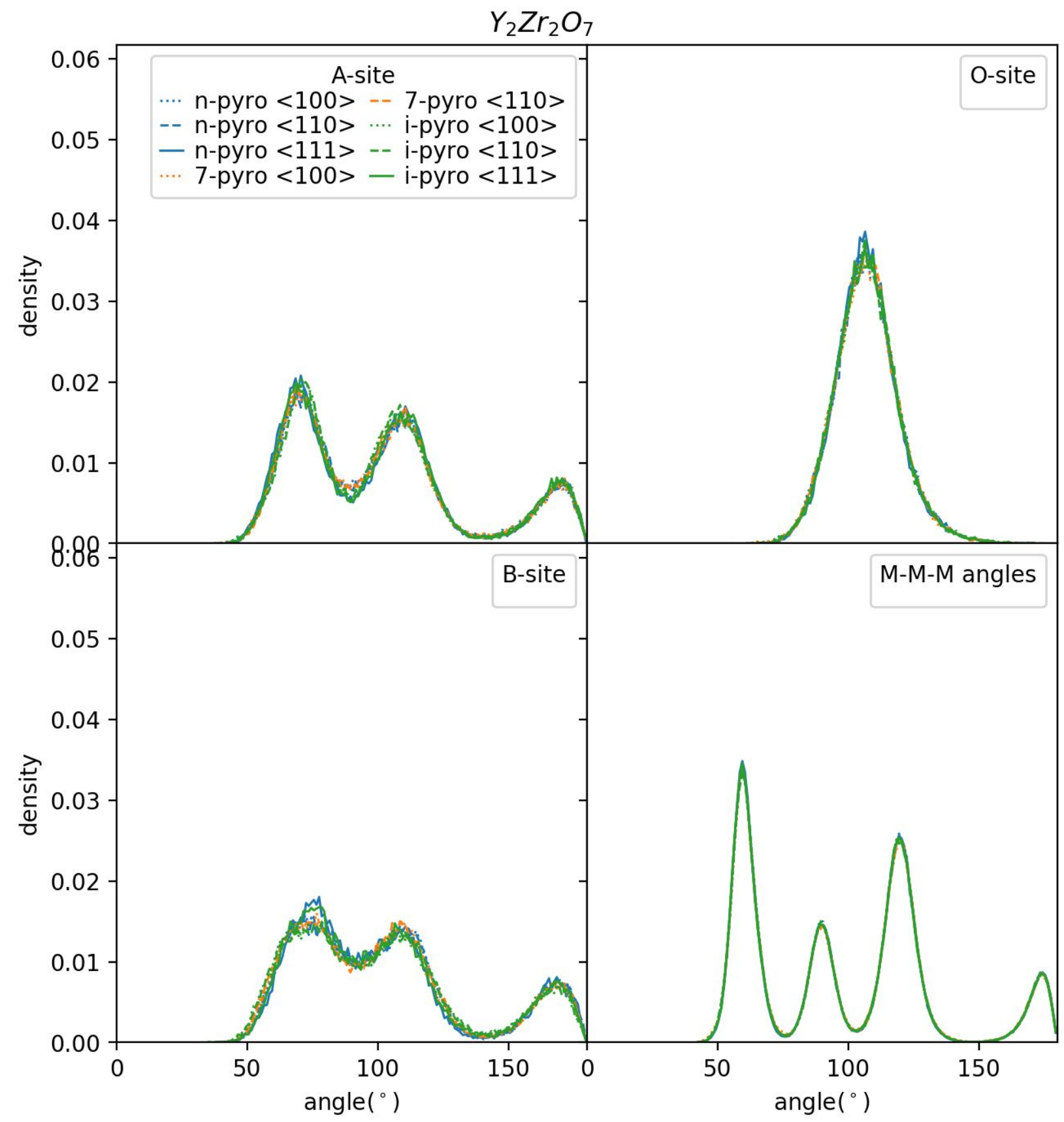

Figure S36: $\mathrm{Y}_{2} \mathrm{Zr}_{2} \mathrm{O}_{7}$ inter-atomic angles extracted from the $\mathrm{RMC}$ refinements. The A-site refers to $\mathrm{O}-\mathrm{A}-\mathrm{O}$ angles, the $B$-site refers to $O-B-O$ angles, the $O$-site refers to $O-M-O$ angles and $M-M-M$ angles refers to all angles between nearest-neighbor cations. The distributions have been overlaid by refinement strategy. 


\section{Polyhedral M-O average and standard deviation}

Figure 37 and Figure S38 show the histograms of the average and standard deviation of the M-O bond lengths surrounding the $A$ - and B-site cations.

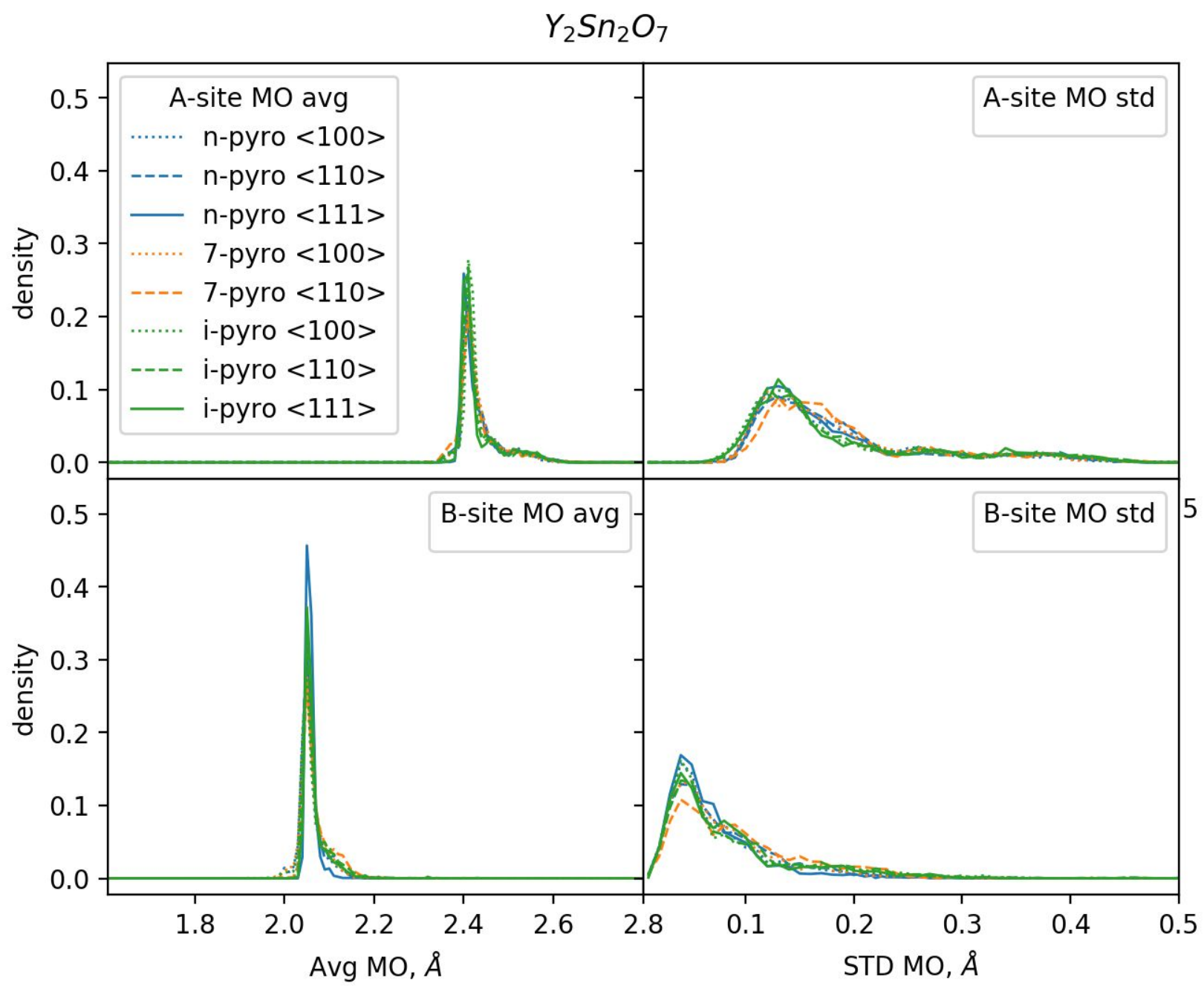

Figure 37: $\mathrm{Y}_{2} \mathrm{Sn}_{2} \mathrm{O}_{7}$ distributions of the average and standard deviation of $\mathrm{M}-\mathrm{O}$ distances surrounding each cation. The distributions have been overlaid by refinement strategy. 


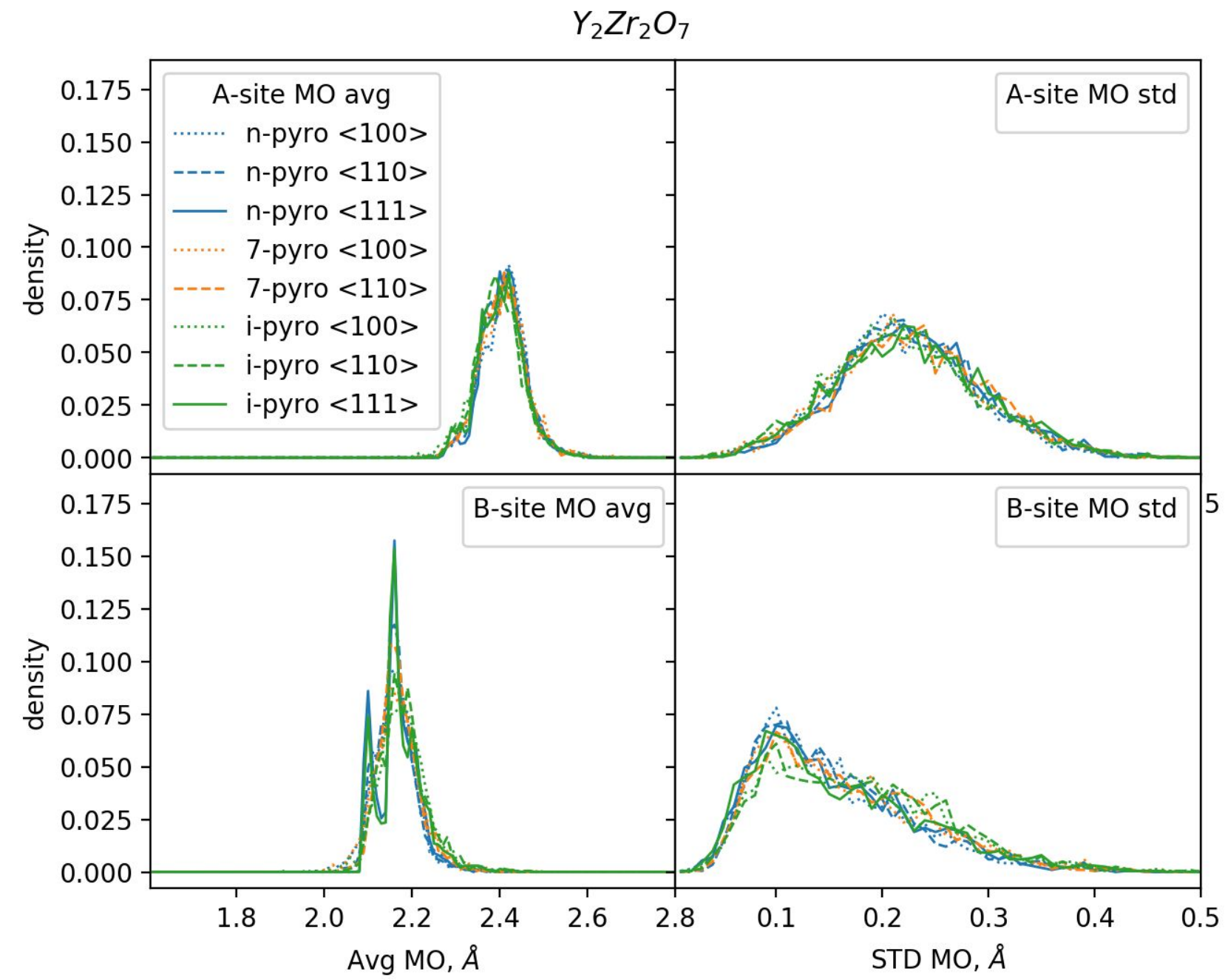

Figure S38: $\mathrm{Y}_{2} \mathrm{Zr}_{2} \mathrm{O}_{7}$ distributions of the average and standard deviation of $\mathrm{M}-\mathrm{O}$ distances surrounding each cation. The distributions have been overlaid by refinement strategy. 


\section{Wyckoff Oxygen site statistics}

Percentages of oxygen in each of the Wyckoff sites.
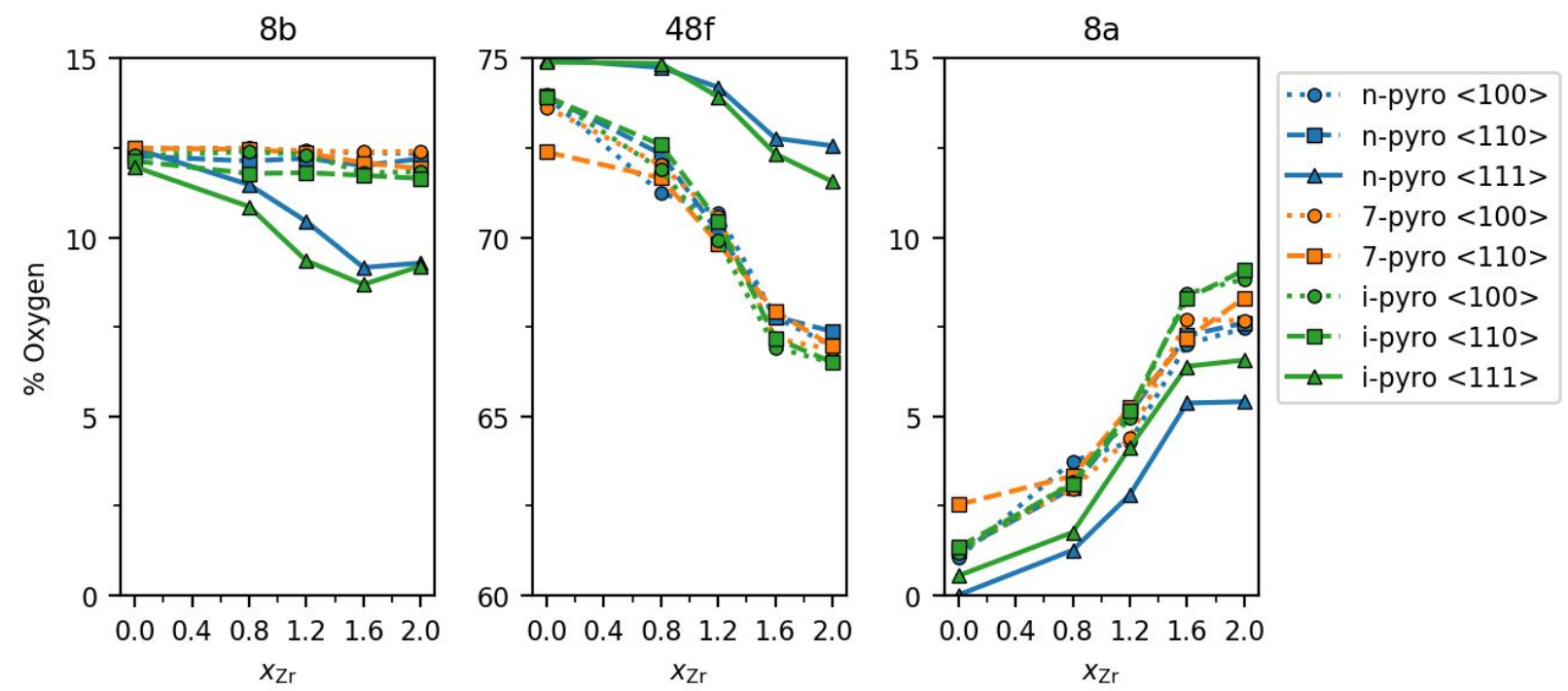

Figure S39: Percentages of total oxygen in the different Fd $\overline{3} m$ Wyckoff sites across the $\gamma_{2} S_{2-x} 2 r_{x} O_{7}$ series, seperated by the 8 refinement strategies that are outlined in the text above. 


\section{Cation coordination statistics}

Percentages of cations in different coordination configurations.

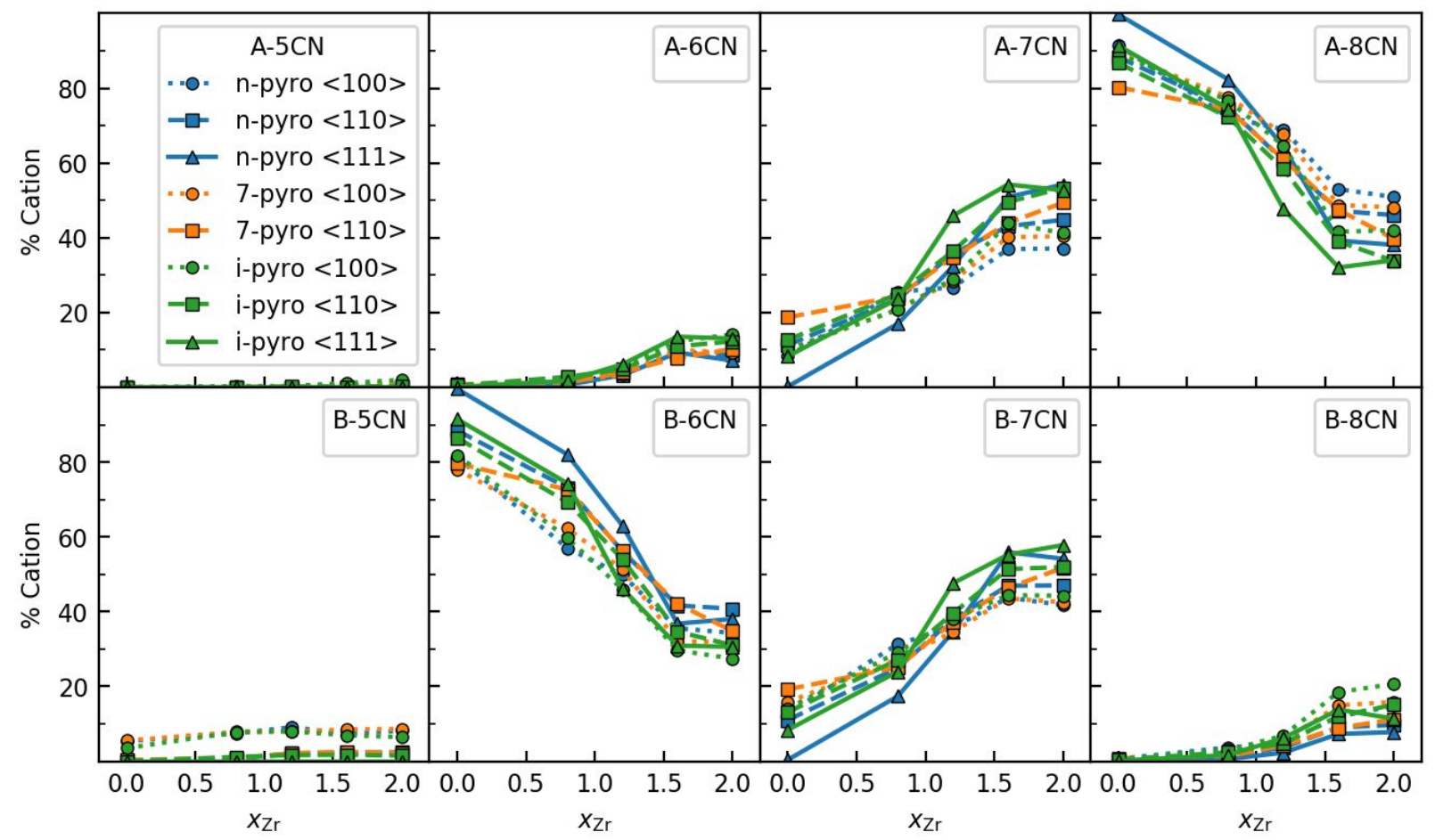

Figure S40: Percentages of cations with different numbers of oxygen coordination across the $\mathrm{Y}_{2} \mathrm{Sn}_{2-x} \mathrm{Zr}_{x} \mathrm{O}_{7}$ series, seperated by the 8 refinement strategies that are outlined in the text above. $\mathrm{CN}=$ coordination number. 


\section{Vacancy nearest neighbor distances for 6-coordinate polyhedra}

Distances between nearest neighbor vacancies around the A- and B-site cations with 6CN.

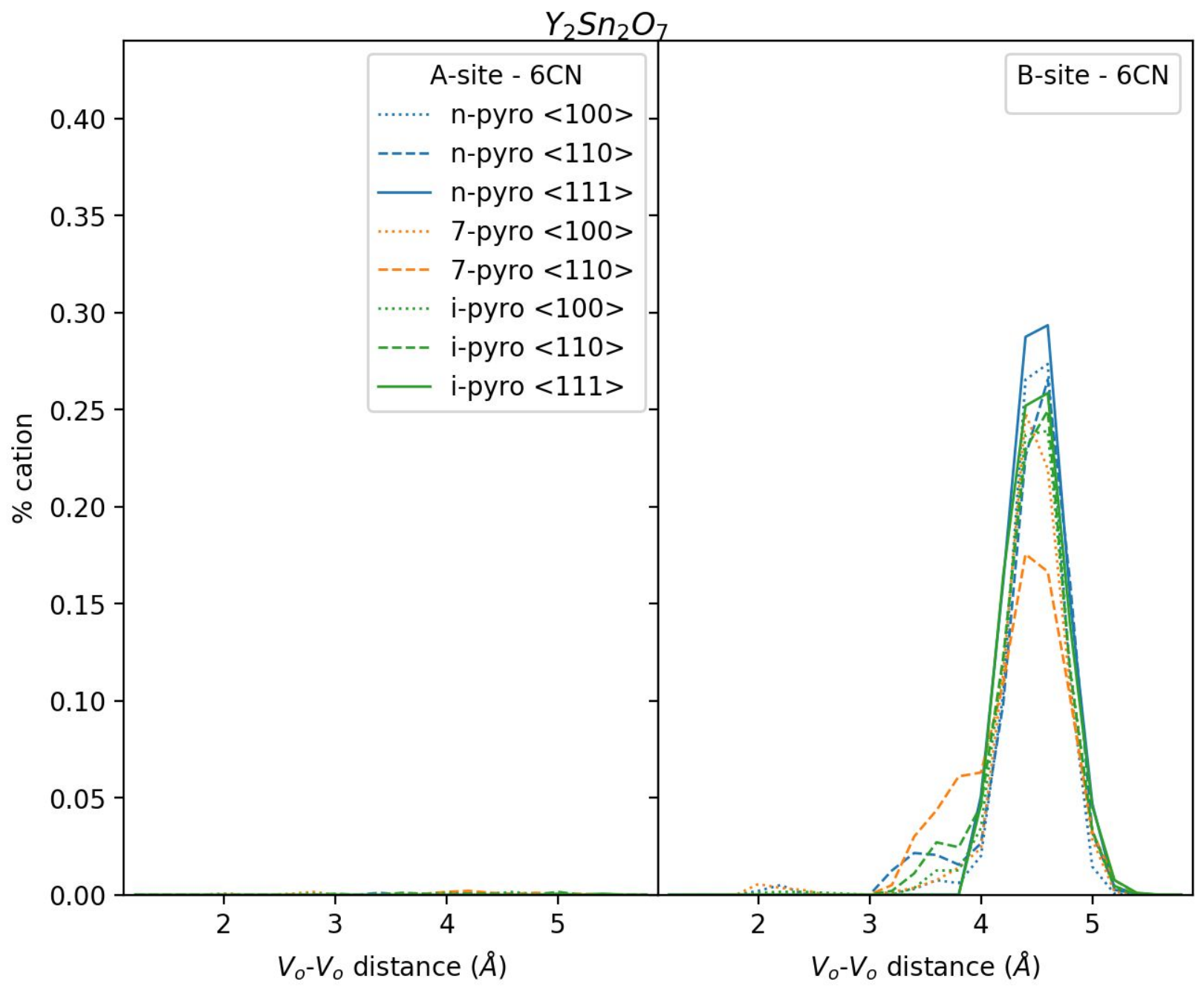

Figure S41: $\mathrm{Y}_{2} \mathrm{Sn}_{2} \mathrm{O}_{7} \mathrm{~V}_{\mathrm{O}}-\mathrm{V}_{\mathrm{O}}$ distances for $6 \mathrm{CN}$ cations, overlaid for each fo the different refinement strategies. 


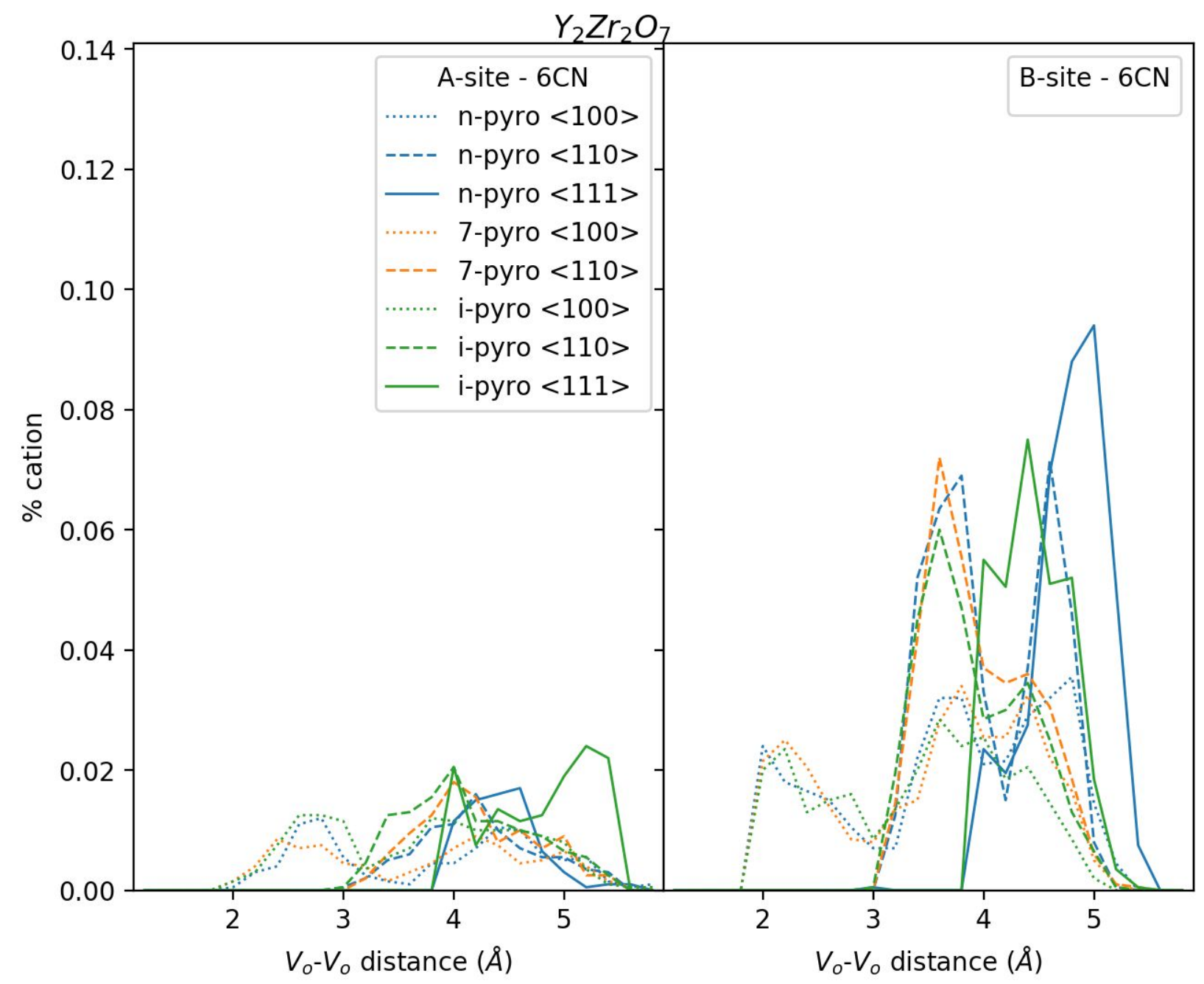

Figure S42: $\mathrm{Y}_{2} \mathrm{Zr}_{2} \mathrm{O}_{7} \mathrm{~V}_{\mathrm{O}}-\mathrm{V}_{O}$ distances for $6 \mathrm{CN}$ cations, overlaid for each fo the different refinement strategies. 


\section{References}

1. Coelho, A., TOPAS and TOPAS-Academic: an optimization program integrating computer algebra and crystallographic objects written in C++. Journal of Applied Crystallography 2018, 51 (1), 210-218.

2. Coelho, A. A.; Chater, P. A.; Kern, A., Fast synthesis and refinement of the atomic pair distribution function. Journal of Applied Crystallography 2015, 48 (3), 869-875.

3. Keen, D., A comparison of various commonly used correlation functions for describing total scattering. Journal of Applied Crystallography 2001, 34 (2), 172-177.

4. Matthew, G. T.; David, A. K.; Martin, T. D.; Andrew, L. G.; Qun, H., RMCProfile: reverse Monte Carlo for polycrystalline materials. Journal of Physics: Condensed Matter 2007, 19 (33), 335218. 